\title{
Directional Responding of C57BL/6J Mice in the Morris Water Maze Is Influenced by Visual and Vestibular Cues and Is Dependent on the Anterior Thalamic Nuclei
}

\author{
Robert W. Stackman Jr, ${ }^{1,2,3}$ Joan C. Lora, ${ }^{3}$ and Sidney B. Williams ${ }^{1}$ \\ ${ }^{1}$ Department of Psychology, ${ }^{2}$ Center for Complex Systems and Brain Sciences, and ${ }^{3}$ Florida Atlantic University/Max Planck Florida Institute Joint Ph.D. \\ Program in Integrative Biology and Neuroscience, Florida Atlantic University, Boca Raton, Florida 33431
}

Recent findings indicate that rats navigate in spatial tasks such as the Morris water maze (MWM) using a local cue-based reference frame rather than a distal cue-based reference frame. Specifically, rats swim in a particular direction to a location relative to pool-based cues, rather than to an absolute location defined by room-based cues. Neural mechanisms supporting this bias in rodents for relative responding in spatial tasks are not yet understood. Anterior thalamic neurons discharge according to the current directional heading of the animal. The contribution of head direction (HD) cell activity to navigation has been difficult to elucidate. We found that male C57BL/6J mice trained for 4 or $7 \mathrm{~d}$ in the MWM exhibited an overwhelming preference for swimming in a direction relative to pool-based cues over absolute responding during a platform-less probe test. Rotation of extramaze cues caused a corresponding rotation of the direction mice swam during the probe test, suggesting that both pool- and room-based reference frames guide platform search. However, disorienting the mice before the probe test disturbed relative responding. Therefore, relative responding is guided by both internal and external cue sources. Selective inactivation of anterior thalamic nuclei (ATN) by microinfusion of muscimol or fluorophore-conjugated muscimol caused a near complete shift in preference from relative to absolute responding. Interestingly, inactivation of the dorsal CA1 region of the hippocampus did not affect relative responding. These data suggest that ATN, and HD cells therein, may guide relative responding in the MWM, a task considered by most to reflect hippocampal processing.

\section{Introduction}

A neural circuit that includes the hippocampus, entorhinal cortex, subicular complex, and anterior thalamic nuclei (ATN) is essential for spatial navigation and path integration in rodents (McNaughton et al., 2006). Spatial navigation is guided by exteroceptive (i.e., landmarks) and interoceptive cues (i.e., vestibular, motor efference copy, etc.). Firing rates of place cells in hippocampus encode the relative spatial location of the animal in an environment (O'Keefe and Nadel, 1978; O'Keefe et al., 1998), while firing rates of head direction (HD) cells in anterodorsal (Taube, 1995) and anteroventral (Yoganarasimha et al., 2006; Tsanov et al., 2011) thalamic nuclei encode relative directional heading of the animal. Neuronal signals representing current lo-

Received Sept. 23, 2011; revised June 2, 2012; accepted June 8, 2012.

Author contributions: R.W.S. designed research; R.W.S., J.C.L., and S.B.W. performed research; R.W.S. and J.C.L. analyzed data; R.W.S., J.C.L., and S.B.W. wrote the paper.

This work was supported by NIH-NIAAA Grant AA14407 (R.W.S.). We thank Shiao-Ying Chow, Michael Guidi, and Christina Clements for assistance with behavioral testing, surgical expertise, and histological procedures, respectively. We thank Dr. Ken Dawson-Scully (Department of Biological Sciences, Florida Atlantic University) for technical assistance acquiring epifluorescence images, and Lisa Rios (Florida Atlantic University) for assisting with manuscript editing.

The authors declare no competing financial interests.

Correspondence should be addressed to Dr. Robert W. Stackman Jr, Department of Psychology, BS 101, Florida Atlantic University, 777 Glades Road, Boca Raton, FL 33431-0991. E-mail: rstackma@fau.edu.

S. B. Williams's present address: University of Alabama Birmingham, Neuroscience Graduate Program, SHEL 1014, 1825 University Boulevard, Birmingham, AL 35294.

DOI:10.1523/JNEUROSCI.4868-11.2012

Copyright $\odot 2012$ the authors $\quad 0270-6474 / 12 / 3210211-15 \$ 15.00 / 0$ cation and directional heading and their integration are influenced by exteroceptive and interoceptive cues and are considered essential information streams that support spatial navigation (Moser et al., 2008). However, the degree to which spatially tuned neuronal activity influences spatial behavior remains unclear. Lesions of ATN impair exteroceptive cue-based navigation by rodents in spatial tasks (Béracochéa and Jaffard, 1994; Aggleton et al., 1996; van Groen et al., 2002; Mitchell and Dalrymple-Alford, 2006; Wolff et al., 2008). Studies in which anterior thalamic HD cells have been recorded while rodents perform a spatial task have yielded little evidence to support the view that HD cells consistently guide spatial behavior (for review, see Muir and Taube, 2002; Stackman, 2010). Examining spatial behavior that requires the animal to attend to directional information and verifying that the brain region, from which HD cells are recorded, is engaged by the spatial task may help to elucidate the relationship of HD cells and spatial behavior. The present study examined these two issues as they apply to the Morris water maze (MWM) and examined the degree to which the ATN and hippocampus support directional behavior.

The MWM is commonly used to assess absolute navigation in rodents, where the goal is defined by its absolute position with respect to distal cues (Morris et al., 1982). A recent review indicated that rats may solve the MWM task using local and distal cue reference frames (Knierim and Hamilton, 2011). This interpretation accounts for the preference of male rats to navigate in a particular direction to a relative location during probe tests in the 


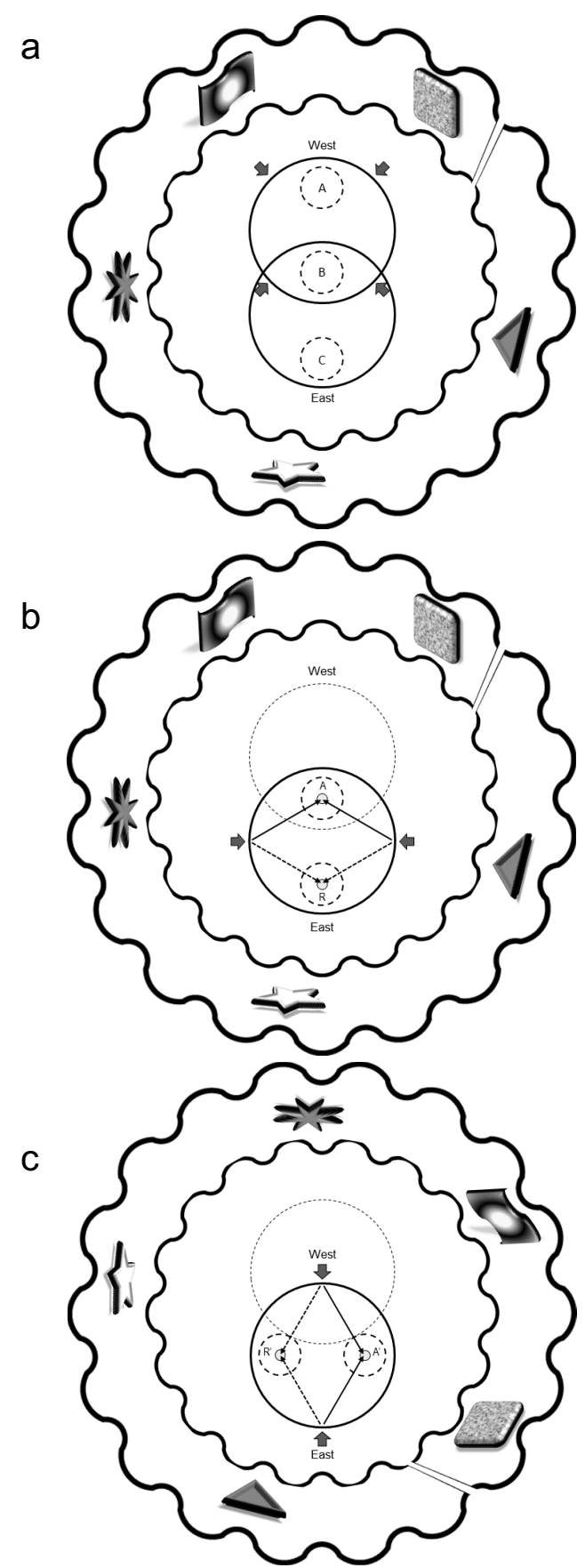

Figure 1. Task design, linear translation of pool, and probe test conditions. $\boldsymbol{a}$, The schematic illustrates the two locations in the testing room where the pool (large black circles, West and East) was positioned during hidden platform training. Respective platform locations, A-C. Mice were trained in the west pool position to platform A or B, and in the east pool position to platform B or C. Gray arrowheads indicate the points where the mice were released into the west pool during training trials. Extramaze visual cues were positioned on a circular black floor-toceiling curtain ( $2.4 \mathrm{~m}$ diameter) that surrounded the pool. The vertical break in the curtain at approximately two $o^{\prime}$ clock represents the point of entry through the curtain. $\boldsymbol{b}$, Before a probe test, the pool was translated linearly from the west position (thin dashed circle) in the testing room to the east position (solid black circle), or vice-versa (schematic not shown). Gray arrowheads indicate the two points (north and south) where the mice were released during the probe test. For mice trained to platform location B (relative vs absolute condition), the translation permitted examining preference for mice to exhibit place or absolute responding [swimming to the absolute search (dashed circle, A) zone during the probe] or relative responding [swimming to the relative search zone (dashed circle, R) during the probe test]. For mice trained to platform location A (relative-only condition), the translation resulted in the trained place being inaccessible during the probe test. Behavior in this condition was characterized by swimming to the
MWM (Hamilton et al., 2007, 2008) as well as in T-maze tasks (Blodgett et al., 1949; Skinner et al., 2003). Compared to rats, mice exhibit modest absolute learning in the MWM, and their spatial responding may be less dependent on extramaze cues (Whishaw, 1995; Whishaw and Tomie, 1996). Here, we verified that male C57BL/6J mice also exhibit a preference for relative responding in the MWM. Rotation of distal landmark cues by $90^{\circ}$ caused relative responding of mice to shift by a similar degree. Disorientation by gentle rotational stimulation disrupted relative responding. Relative responding of mice in MWM was disrupted after temporary inactivation of ATN, but intact after inactivation of dorsal hippocampus. These results substantiate the view that the ATN, and perhaps activity of HD cells therein, are critical for the use of directional information in a navigation task.

\section{Materials and Methods}

Mice

Subjects were male 8- to 12-week-old C57BL/6J mice purchased from the Jackson Laboratories. Mice were housed four per standard polycarbonate cage with ad libitum access to food and water. Cages were maintained in a temperature- and humidity-controlled vivarium with a $12 \mathrm{~h}$ light/ dark cycle (lights on at 7:00 A.M.). All behavioral testing took place during the lights on phase of the cycle (specifically, from 11:00 A.M. to 3:00 P.M.). All mice were habituated to the Florida Atlantic University vivarium for at least $5 \mathrm{~d}$ before the start of experiments. All procedures were conducted in accordance with the guidelines described in the $\mathrm{Na}$ tional Institutes of Health Guide for the Care and Use of Laboratory Animals. The Florida Atlantic University Institutional Animal Care and Use Committee approved all procedures. A naive cohort of C57BL/6J mice was used for each of the experiments described below.

\section{Morris water maze}

Training. The pool (109 $\mathrm{cm}$ diameter, $65 \mathrm{~cm}$ high), constructed of seamless white polyethylene, was placed on a square plywood platform with appliance rollers attached to the bottom for easy linear translocation. The clear plastic escape platform ( $8 \mathrm{~cm}$ diameter, $31 \mathrm{~cm}$ high) could be positioned in the center, east, or west position in the pool. The pool was filled to $1 \mathrm{~cm}$ above the platform with water rendered opaque by stirring in nontoxic white tempura paint. The water temperature was maintained at a range of $22-24^{\circ} \mathrm{C}$. A circular black curtain around the pool eliminated competing environmental cues. Behavior of the mice in the pool was recorded by a video camera positioned on the ceiling in the center of the testing room. The video camera was interfaced with the EthoVision 3.1 computerized video-tracking system (Noldus Information Technologies), which permitted the off-line analysis of multiple behavioral parameters. Mice, in squads of four, were removed from their home cage and transported into the testing room inside an empty cage from an adjacent room. Mice received $2 \mathrm{~d}$ of nonspatial training (one trial per day) to acclimate to the pool and the submerged platform, and then received hidden platform training (four trials per day) to examine hippocampaldependent spatial learning and memory (Morris et al., 1982). During nonspatial training, the pool was moved to the center of the testing room, and the platform was positioned in the center of the pool and submerged $1 \mathrm{~cm}$ below the water surface. Each nonspatial training trial comprised of an experimenter retrieving a mouse from the holding cage, carrying it inside the curtained area, and placing the mouse onto the platform for $60 \mathrm{~s}$. Afterward, the mouse was released into the pool four times (at

relative search zone or to the opposite search zone. Search behavior was quantified by a heading error score for each probe test, which was computed by plotting the initial swim path from release to arrival into the relative search zone or relative/opposite search zone and measuring the angular deviation from a direct, straight path to the relative search zone (dashed lines). $c$, For the rotation of visual cues and rotation of visual cues plus translation of the pool conditions, the extramaze cues were rotated $90^{\circ}$ clockwise before the probe test in the translated pool (no pool translation in the former condition). The entry point through the curtain, the two release points, and the search zones of interest, $R^{\prime}$ and $A^{\prime}$ were also rotated $90^{\circ}$ clockwise. 
north, south, east, and west positions adjacent to the platform) to practice swimming and climbing onto each platform. During hidden platform training, the pool and platform were positioned in one of two locations: 40 mice were trained with the pool in the west side of the testing room (Fig. 1A). For a second cohort of 40 mice, the pool was positioned in the east side of the testing room. There were three possible platform locations used relative to the extramaze cues (Fig. $1 \mathrm{~A}$, diagram): position A (west side of the pool when positioned in the west), B (east side of pool when positioned in the west, and west side of the pool when positioned in the east; Fig. $1 B$, diagram), and $\mathrm{C}$ (east side of the pool when positioned in the east). The platform location remained fixed in one of these three positions throughout hidden platform training. Several large three-dimensional visible cues [a white lab coat; a square cardboard sheet colored hot pink and having jagged edges $(0.5 \times 0.5 \mathrm{~m})$; a multicolored lei; two baseball hats clipped together; an elongated, brightly designed cardboard box $(0.1 \times 0.1 \times 1.0 \mathrm{~m})$; and a $2 \mathrm{~m}$ black steel torchiere lamp with a white shade] were attached to the circular curtain surrounding the pool, which provided landmarks to the mice during hidden platform training. During a hidden platform trial, the experimenter removed the mouse from its holding cage outside the curtained area, carried it on his or her arm inside the curtained area, and released it into the pool facing the pool wall. Release points (Fig. $1 \mathrm{~A}$, arrowheads) around the edge of the pool (northeast, southeast, southwest, and northwest) were randomized each day. The mouse was allowed $60 \mathrm{~s}$ to reach the hidden platform, after which it was guided to the platform. The mouse remained on the platform for $30 \mathrm{~s}$ to view spatial cues, and was then returned to the holding cage outside of the curtained enclosure. The experimenter then retrieved the next mouse of that given squad for its training trial. All of the mice in a squad completed one trial before the second trial was conducted. In this manner, the intertrial interval was $\sim 3 \mathrm{~min}$. After the last training trial of each day, the mice were placed under a warm air stream for several minutes and were then returned to their home cages. Each mouse received 28 training trials on the hidden platform water maze task. Spatial learning was measured by computing daily means for escape latency (in seconds) and the cumulative distance to the platform (in centimeters) over $7 \mathrm{~d}$ of training and analyzing these dependent measures with a repeated-measures ANOVA.

Relative vs absolute probe test. A total of 80 mice were trained in the water maze as described above and then received a probe test to determine whether male C57BL/6J mice exhibit relative responding during spatial memory retention testing. Each mouse received a probe test immediately after the final training trial on the seventh day. Before presenting the probe test, the platform was removed from the pool, and the pool was translated linearly in the room to a position opposite of that during training for 60 mice, (i.e., translated east for mice trained with the pool in the west location) (Fig. 1B). For the remaining 20 mice, the same pool position was used for the probe as during training. Thus, there were three distinct probe test conditions: relative versus absolute, relative only, and no shift. Mice were randomly assigned to one of these three conditions. As depicted in Fig. 1 B, mice in the relative versus absolute condition were those that were trained to locate the platform at position B. When the pool was translated, the absolute location where the platform was found during training, as defined by the extramaze cues (i.e., absolute), was now in the relative opposite location of the pool. Thus, if during the probe test the mice chose to swim directly to the previous place (in the room) where the platform was located during training, then, in the translated pool, they would actually swim in a direction opposite that used to locate the platform during training. If the mice chose to swim in the same relative direction from the release point in the translated pool, then they would arrive in a pool location opposite that used during train. Thus, linear translation of the pool permitted assessing the cues and responses that supported platform search during the probe test. The purpose of the probe test was to verify that C57BL/6J mice exhibit a preference for relative responding over absolute responding in the water maze, as had been reported for male rats (Hamilton et al., 2007). It was predicted that mice in the relative versus absolute condition (Fig. $1 B$ ) would either swim to the absolute goal location based on the distal visual cues using absolute navigation (absolute), or swim to the relative goal location in the pool by directional responding (relative). For the relative-only condi- tion, the absolute goal location was beyond the pool wall after the translation of the pool. For the probe tests, mice were released into the pool at novel points (north and south) that were equidistant from the relative location and the absolute location (Fig. $1 B$, arrowheads). After completion of the probe test, mice were placed under the warm air stream and then returned to their home cages. From the 30 s probe test, the following measures were analyzed: the latency to first enter, and the dwell time in, circular zones centered on the relative and absolute locations. These circular search zones were three times the diameter of the platform (Fig. $1 B$, dashed circles). The percentage of the trial spent in each of the four pool quadrants was also measured. To further quantify relative responding, the angle of the mouse's swim path from the release point to the first crossing into either the relative (R) or absolute (A) zone was plotted. A heading error (in degrees) was then computed for each probe test by subtracting the angle of the actual path from the angle of a direct path from the release point directly to the relative zone.

Influence of extent of training on relative vs absolute responding. To determine whether the relative responding seen in the cohort of mice trained for $7 \mathrm{~d}$ in Experiment 1 occurs at earlier stages of training or was due to overtraining, naive cohorts of 12 naive male C57BL/6J mice were trained in the MWM for four trials per day for just 2 or $4 \mathrm{~d}$ and then probed in the translated pool. Other than the number of training trials, all other procedures were as described for Experiment 1.

Rotation of visual cues. A naive cohort of 12 male C57BL/6J mice was trained in the MWM for four trials per day for just $4 \mathrm{~d}$ with the pool in the west position and the platform in position $\mathrm{B}$, as described above. A probe test was imposed after the last training trial on the fourth day to examine whether platform search behavior in C57BL/6J mice early in training is influenced by the distal visible cues. The pool was not shifted from the west position, and the black curtain, containing all of the distal visual cues, was rotated $90^{\circ}$ clockwise. Each mouse was introduced into the pool at the west or east point. Initial search paths were analyzed by computing the angle of the path from the release point to the first crossing into either the A zone or the zone in the opposite location in the pool [opposite $(\mathrm{O})$ zone]. As above, a heading error (in degrees) was computed by subtracting the angle of the actual path from the angle of a direct path from the release point directly to the absolute zone.

Rotation of visual cues plus linear translation of the pool. A naive cohort of 12 male C57BL/6J mice were trained in the MWM with the pool in the west position and the platform in position $B$, as described above. All mice were trained for four trials per day for $7 \mathrm{~d}$. A probe test was imposed after the last training trial on the seventh day to examine whether the distal visible cues exert stimulus control over relative responding. The pool was translated from the west position to the east position, and the black curtain, containing the distal visual cues, was rotated $90^{\circ}$ counterclockwise (Fig. $1 C$ ). All other procedures were conducted as described above, except that the experimenter entered the curtained enclosure at a position $90^{\circ}$ shifted counterclockwise from the original position. The mouse was released into the pool at the west or east point. For the purposes of analyzing relative responding, we again calculated heading error, but for this condition, the direct relative path (i.e., $0^{\circ}$ error) was shifted $90^{\circ}$ clockwise from that used to analyze relative behavior in the probe tests of Experiment 1; that is, if a given mouse first swam in a straight path directly from the release point to the rotated relative $\left(\mathrm{R}^{\prime}\right)$ search zone as opposed to the rotated absolute $\left(\mathrm{A}^{\prime}\right)$ search zone, then its heading error score would be $-90^{\circ}$.

Disorientation. On the day following the "rotation of visual cues plus linear translation of the pool" probe test, the pool was returned to its original position in the room, and mice were retrained to the original platform position (B) for $2 \mathrm{~d}$ (four trials per day). After the final retraining trial, another $30 \mathrm{~s}$ probe test was conducted to examine the influence of pretest rotational stimulation on relative responding. The pool was translated from the west position to the east position, and the platform was removed from the pool. Each individual mouse was removed from the holding cage and placed into a corrugated cardboard box $(22 \times 15 \times$ $15 \mathrm{~cm}$ ) containing corncob bedding. The top flaps of the box were closed, and then the box was gently rotated clockwise and counterclockwise for $60 \mathrm{~s}$ as the experimenter (R.W.S.) walked slowly around the pool. The rate of rotation of the box varied but was estimated at $\sim 90-180^{\circ}$ per 
second. After $60 \mathrm{~s}$, the box was returned to the counter outside of the curtained enclosure, and the box remained closed for another $30 \mathrm{~s}$. Afterward, the box was picked up and carried into the curtained enclosure, and the mouse was removed from the box and released into the pool at the north or south point (Fig. $1 B$ ). All mice seemed to tolerate the disorientation procedure, and none of the mice exhibited any difficulty swimming after this manipulation. All other procedures were identical to that described above.

\section{Temporary inactivation of anterior thalamus or CA1 region of dorsal hippocampus}

Stereotaxic surgery. A naive cohort of male C57BL/6J mice underwent stereotaxic surgery during which chronic bilateral intracranial guide cannulae (26 gauge; Plastics One) were implanted directly above the anterodorsal thalamic nuclei or above the dorsal CA1 region of the hippocampus. Mice were anesthetized with isoflurane (3-5\% to induce; $1 \%$ for maintenance) via a VetEquip vaporizer. Coordinates of guide cannulae (from bregma) were as follows: for anterodorsal thalamic nuclei, $0.80 \mathrm{~mm}$ posterior, $\pm 0.75 \mathrm{~mm}$ lateral, and $1.75 \mathrm{~mm}$ ventral to the cortical surface; for dorsal CA1, $2.0 \mathrm{~mm}$ posterior, $\pm 1.5 \mathrm{~mm}$ lateral, and $1.6 \mathrm{~mm}$ ventral to the skull surface (Franklin and Paxinos, 2008). Guide cannulae were held in place with two $1 / 8$ inch stainless-steel jeweler's screws (000-120; Small Parts) and cold-curing acrylic dental cement. Dummy cannulae (9.1 mm; Plastics One) were inserted into each guide cannula to prevent blockage and held in place with fitted dust caps (Plastics One). Immediately after the surgery, mice received an intraperitoneal injection of $0.8 \mathrm{ml}$ of sterile $0.9 \%$ saline to reduce dehydration from the surgery. Each mouse also received buprenorphine $(0.05 \mathrm{mg} / \mathrm{kg}$, s.c. $)$ every $6-12 \mathrm{~h}$ after surgery for three injections. Mice were returned to their home cage upon recovery of the righting reflex. Water maze training was not initiated until a 2 week postoperative recovery period was complete.

Intracranial microinfusion. After postoperative recovery, all mice received $7 \mathrm{~d}$ of hidden platform water maze training (four trials per day) with the pool positioned in the west of the testing room. A probe test was presented after the last training trial, as described above with the only exception being that each mouse received an intracranial infusion into the anterodorsal thalamus or the CA1 region before testing. Before the probe test, each mouse was gently restrained, the dust cap and dummy cannulae removed, and bilateral infusion cannula inserted through the guide cannulae. Each mouse was then released into a holding cage where it received a $0.25 \mu \mathrm{l}$ infusion (at a rate of $0.167 \mu \mathrm{l} / 60 \mathrm{~s}$ for $90 \mathrm{~s}$ ) of either artificial CSF [aCSF; containing (in $\mathrm{mm}$ ) $147 \mathrm{NaCl}, 2.9 \mathrm{KCl}, 1.6 \mathrm{MgCl}_{2}$, $1.7 \mathrm{CaCl}_{2}, 2.2$ dextrose, $35.9 \mathrm{NaHCO}_{3}, \mathrm{pH} 7.4$ ] or muscimol $(0.2 \mu \mathrm{g}$; Tocris Bioscience) infused by two $10 \mu$ l gas-tight Hamilton syringes held in a CMA/400 microinfusion pump (CMA Microdialysis). The infusion cannulae remained in place for $2 \mathrm{~min}$ after completion of the infusion to permit diffusion. Each mouse was again gently restrained, the infusion cannulae withdrawn, and sterile dummy internal cannulae inserted and dust caps replaced. Each mouse was then placed into a clean holding cage for $10 \mathrm{~min}$ before being transported into the water maze testing room for the probe test. All probe tests for this experiment were conducted with the pool linearly translated to the east position in the testing room-the relative versus absolute condition or the relative-only condition (Fig. $1 B$, diagram). After completion of the probe test, mice were placed under the warm air stream and then returned to their home cages. On each of the following $2 \mathrm{~d}$, each mouse received four hidden platform trials with the pool in the west position of the room. Each mouse received a second intracranial microinfusion and a probe test after the fourth trial on the second day. Treatment assignments were reversed for the second microinfusion (i.e., a within-subjects crossover design) so that each mouse received both treatments.

Microinfusion of fluorophore-conjugated muscimol. To appreciate the degree of tissue distribution of microinfused muscimol associated with behavioral effects, a naive cohort of male C57BL/6J mice was implanted with guide cannulae directed at the anterodorsal thalamic nucleus $(n=$ 7 ) or at the CA1 region of dorsal hippocampus $(n=9)$ and then trained in the water maze as above. These mice received bilateral intracranial microinfusions of fluorophore-conjugated muscimol (FCM; $0.2 \mu \mathrm{g} / 0.25$ $\mu l$; BODIPY TMR-X; Invitrogen) prepared in PBS or PBS vehicle just before a probe test in the translated pool. The mice received eight more training trials over the next $2 \mathrm{~d}$ and then received a second probe test after the last training trial. As above, the treatment assignments were reversed for the second probe test (i.e., mice that previously received PBS vehicle now received FCM and vice versa). The probe test was presented after a $10 \mathrm{~min}$ delay. Three days after the final probe test, all mice received a second microinfusion of FCM, and 20 min later the mice were overdosed with a ketamine/xylazine mixture and were perfused transcardially with $0.9 \%$ saline followed by $4 \%$ paraformaldehyde. Brains were sectioned at $40 \mu \mathrm{m}$ on a Leica vibratome and floated in PBS before mounting on chrom-alum gelatin-coated glass slides.

Histological analysis. Mice were killed with an overdose of isoflurane. The brains were gently removed and placed in a $4 \%$ paraformaldehyde in a PSB solution ( $10 \mathrm{~mm}[\mathrm{SCAP}]), \mathrm{pH} 7.4$, for a minimum of $24 \mathrm{~h}$. Each brain was cryoprotected by equilibrating it in a $20 \%$ and then $30 \%$ sucrose solution (in PBS). All brains were then sectioned $(40 \mu \mathrm{m})$ at $-19^{\circ} \mathrm{C}$ on the freezing stage of a Leica sliding microtome (model SM2010R), mounted, and stained with cresyl violet. Cannulae placement was analyzed using a Nikon Eclipse 55i light microscope, and images were captured using NIS-Elements D on a Nikon DS-Fil digital camera. For a subset of mice, cresyl violet stain was injected $(0.25 \mu \mathrm{l} /$ side $)$ into the ATN right before killing to facilitate detection of the microinfusion site from the brain sections. Histological analysis was performed as described previously, except the tissue was stained with Neutral red to better visualize the site of microinfusion. Data from mice $(n=6)$ with incorrect cannulae placement were excluded from the analysis.

Fluorophore distribution. To estimate the distribution of FCM after microinfusion, photomicrographs were taken to capture the injection sites and the anterior-posterior spread of FCM. The fluorescence images were scanned with a Nikon C1si Fast Spectral Confocal system with an acousto-optic tunable filter laser unit (Nikon) using $4 \times$ and $10 \times$ objectives. Each sample was scanned at an image resolution of $2048 \times 2048$ pixels at $1 \times$ zoom. Digital images were captured using Nikon Elements, and then exported into Adobe Photoshop to adjust brightness and contrast. A subset of ATN sections was first counterstained with a green fluorescent Nissl stain (NeuroTrace; Invitrogen) before images were captured for analysis to better identify boundaries of brain regions surrounding the injection sites. In these cases, images of the FCM and the fluorescent Nissl were reassembled into the red and green color channels, respectively, in Elements or Photoshop.

\section{Statistical methods}

Data from the water maze training trials were analyzed as four-trial block means with a one-, two- or three-factor ANOVA with trial block as the repeated measure. For the probe test data, one-factor ANOVAs or Student's $t$ tests were conducted to determine significant preference for a given pool quadrant or a preference for search zone choice, respectively. Two within-factors (treatment and search zone) ANOVAs were conducted to test the influence of region-specific muscimol-induced inactivation on relative responding. Bonferroni tests were used for post hoc comparisons. Furthermore, $\chi^{2}$ tests were used to analyze the frequency with which mice exhibited relative or absolute responding during the probe tests. For the $\chi^{2}$ analyses, a conservative estimate of the expected frequency of absolute responding was set at $50 \%$. Rayleigh tests of uniformity were run on distributions of circular data (i.e., angles of heading error). For all tests, differences were considered significant at $p<0.05$.

\section{Results}

\section{C57BL/6J mice exhibit a preference for relative over absolute} responding in the Morris water maze

\section{Training}

Mice were trained for 28 trials in the hidden platform water maze task with the pool located in either the west or the east of the testing room. Acquisition of the spatial task was determined by measuring the latency to find the platform and the cumulative distance traveled before reaching the platform. The respective data were averaged for 
a
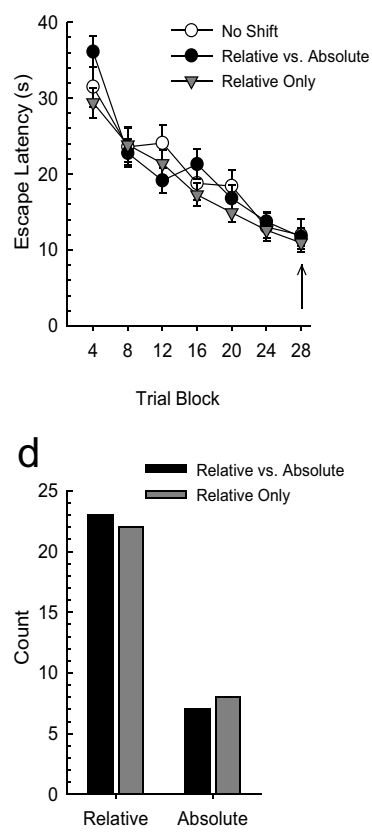

Search Preference b

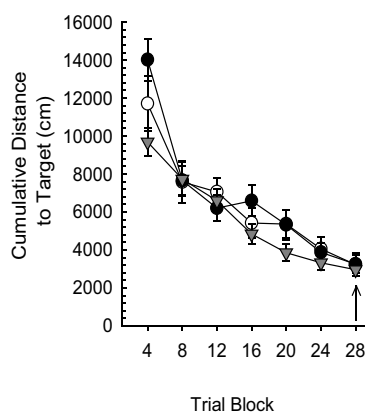

e

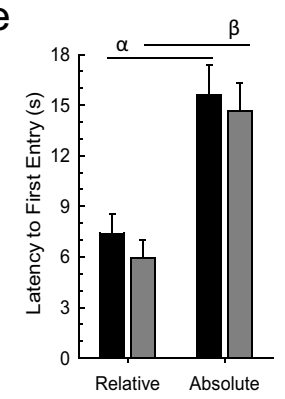

C

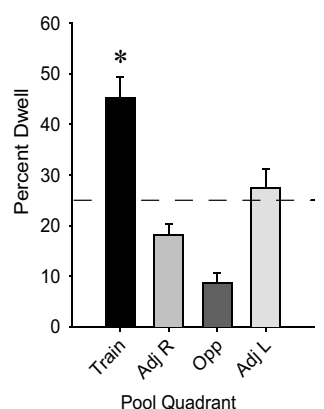

$f$

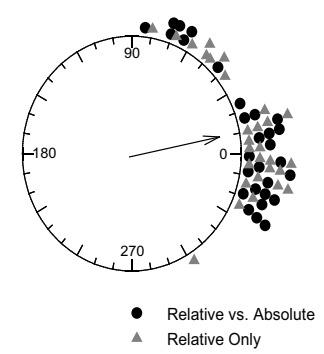

Search Zone

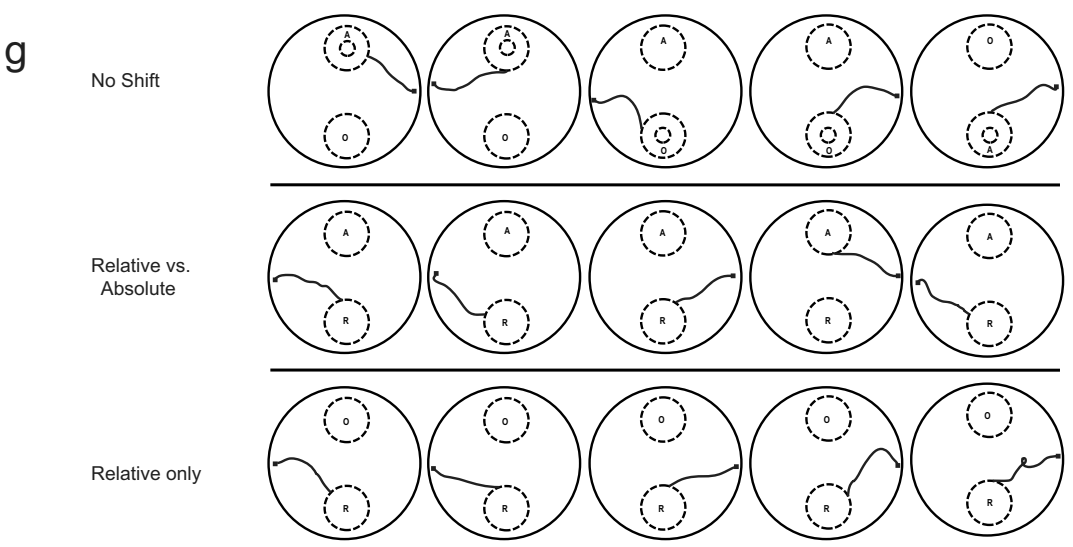

Figure 2. Absolute navigation training and performance of mice under the three probe test conditions (no shift, relative vs absolute, and relative only). $\boldsymbol{a}, \boldsymbol{b}$, The three groups acquired the task equivalently as illustrated by trial block plots of escape latency (a) and cumulative distance to platform center $(\boldsymbol{b})$. The arrow indicates when the probe test was imposed. $\boldsymbol{c}$, No-shift (open circle) mice exhibited a strong preference for searching for the platform in the trained quadrant during the probe test $\left({ }^{*} p<0.01\right.$ vs other quadrants), indicating that all mice acquired the absolute memory for the platform location. $\boldsymbol{d}$, Mice of the relative versus absolute (closed circle) and relative-only (gray triangle) conditions swam first to the relative search zone during the probe test in the translated pool instead of the absolute/opposite search zone. $\boldsymbol{e}$, The mice also exhibited a significantly shorter latency to first enter the relative search zone compared to the absolute/opposite search zone $\left({ }^{\alpha} p<0.001 ;{ }_{p}{ }_{p}<0.001\right)$. $\boldsymbol{f}$, The polar plot of the distribution of individual heading error measures (black circles, relative vs absolute condition; gray triangles, relative-only condition) indicates that the majority of error scores were clustered at $\sim 10^{\circ}$, corresponding to relative responding, and a minor cluster of error scores at $\sim 60^{\circ}$, corresponding to absolute responding. The arrow denotes the combined mean vector angle of $10.3^{\circ}$ for both conditions. The length of the arrow represents the mean vector length $(r=0.86)$ that denotes the variability in heading error scores, with no variability indicated by $r=1 . \boldsymbol{g}$, Representative initial swim paths are depicted for mice of the no-shift (top), relative versus absolute (middle), and relative-only (bottom) conditions. For the no-shift mice, the location of the trained platform $\mathrm{A}, \mathrm{B}$, or $\mathrm{C}$ is depicted; the relative position of the platform during the training trials for these mice is indicated by the small dashed lined circle inside the larger dashed circles that indicate the respective search zones. For the relative versus absolute and relativeonly mice, the respective search zones are indicated by R (relative), A (absolute), and 0 (opposite). Error bars indicate SEM.

each day and analyzed by a repeated-measures ANOVA with training day (Day 1-7), pool position (west or east), and platform location $(\mathrm{A}, \mathrm{B}$, or $\mathrm{C})$ as factors. There was a significant main effect of training day on measures of escape latency $\left(F_{(6,456)}=50.63, p<\right.$ 0.001 ; Fig. $2 A)$ and of cumulative distance to platform center $\left(F_{(6,456)}=60.84, p<0.001 ;\right.$ Fig. $\left.2 B\right)$. There were no significant effects of pool position or platform location, nor were there any significant interactions of day by platform or day by pool position (all $p$ values $>0.05$ ). Mice were then assigned to one of the three probe test conditions (relative vs absolute, relative only, and no shift) to achieve matched groups. Accordingly there was no significant main effect of future probe test condition on escape latency $\left[F_{(2,77)}=1.19\right.$, not significant (n.s.) $]$ or cumulative distance measures $\left(F_{(2,77)}=\right.$ 2.14, n.s.). These results indicate that all mice were matched in terms of spatial task performance regardless of the absolute location of the platform relative to the extramaze cues.

\section{Probe test}

During the $30 \mathrm{~s}$ probe test, all 20 mice in the no-shift condition swam first to the region of the pool that previously contained the platform compared to a similar sized region in the opposite position in the pool $\left(t_{(19)}=\right.$ $-4.01, p=0.001)$. As expected, the mice also spent significantly more time in the previously correct location compared to the opposite position $\left(t_{(19)}=5.72, p<0.001\right)$. Analysis of the percentage of quadrant dwelling of the no-shift mice yielded a significant effect of quadrant $\left(F_{(1,19)}=31.31\right.$, $p<0.001)$. Post hoc Bonferroni multiple comparisons tests revealed that the no-shift mice exhibited a typical preference for searching in the training quadrant of the pool over all three other quadrants (Fig. $2 C$ ). These results are included here as support for the contention that all of the mice had acquired and retained memory for the trained location of the submerged platform in the water maze. Representative swim paths shown in top panel of Figure $2 G$ depict the initial path of five no-shift condition mice from the release point to the first entry into the previously correct search zone (marked A, B, or C) during the probe test.

The probe tests for the remaining mice were conducted after the pool was linearly translated in the testing room. Regardless of pool position, all of the mice exhibited remarkably similar behavior during the $30 \mathrm{~s}$ probe test. Specifically, all of the mice tended to swim immediately from the release point directly toward one of the predetermined search zones. Given the consistency of the quantitative measures of probe test performance across the three conditions described below, it appears that the search behavior of the mice was not impaired by the translation of the pool. For the mice in the relative versus absolute probe test condition, the pool was linearly translated in the testing room before the probe test (e.g., from the west to the east or vice versa). During the probe test, these relative versus absolute condition mice could 
swim to the previously correct location (i.e., absolute search zone), or to swim to the relative location that would have corresponded with the correct location of the platform during the training sessions (i.e., the relative search zone). The relative versus absolute mice swam first to the relative search zone rather than to the absolute search zone $\left(\chi^{2}=8.53, p<0.01\right.$; Fig. $2 D)$; that is, rather than navigating to the absolute place in the room as expected given their prior training and as dictated by the extramaze cues (i.e., absolute), these mice first entered the relatively correct location $\left(t_{(29)}=-3.51, p=0.001\right.$; Fig. 2E), and these mice spent significantly more time in this location than the absolute location $\left(t_{(29)}=2.45, p<0.025\right)$. A search path that was deliberate and direct represented the behavior of the relative versus absolute mice, similar to that of the no-shift mice. Specifically, they left the release point and swam in a relatively straight path that would have taken them directly to the region of the pool where the platform had been located, had the pool not been translated relative to the extramaze cues. Five representative swim paths are shown in the middle panel of Figure $2 G$. Four of these paths clearly illustrate the relative responding of the relative versus absolute mice during the probe test, while the remaining path shows that of a mouse that swam first to the absolute search zone.

The pool was also translated linearly in the testing room before the relative-only probe test condition. The relative-only mice also swam to the relative location in the pool where the platform had been found during training, (i.e., the relative search zone) first before navigating to the region opposite to the relative location. These mice had been trained to find the platform in a location of the testing room that was no longer available to the mice after the pool had been shifted linearly in the testing room. Even so, these mice exhibited a significant preference for swimming first to a region of pool that was in a location directionally consistent with the trained location relative to the release point $\left(\chi^{2}=6.53, p<\right.$ 0.01; Fig. $2 D$ ). Specifically, these mice first entered the relative search zone, or the directionally correct location $\left(t_{(29)}=-3.69\right.$, $p=0.001$, Fig. $2 D, E$ ), and these mice spent significantly more time in this location than the opposite location $\left(t_{(29)}=2.64, p<\right.$ $0.015)$. Here again, examination of the swim paths presented in the bottom panel of Figure $2 G$ reveal that the relative-only mice exhibited relative responding. Consistent with the responses of the relative versus absolute mice, they swam in an approximately straight path in a direction equivalent to that that would have taken them from the release point to the platform had the pool not been shifted relative to the extramaze cues.

One-way ANOVAs revealed no significant effect of probe test condition on the latency to enter the relative search zone of the $\operatorname{pool}\left(F_{(2,79)}=0.5\right.$, n.s. $)$, the latency to enter the opposite zone of b

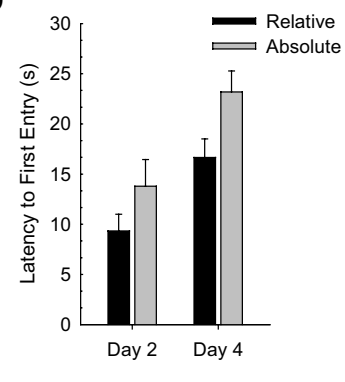

C

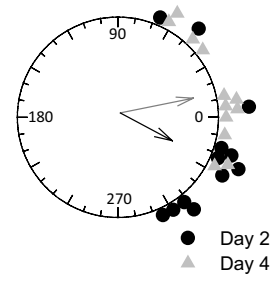

e

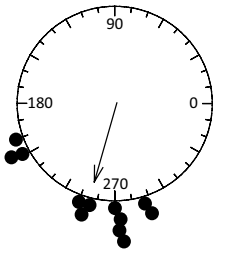

Trial Block

Figure 3. Evidence of absolute and relative responding in mice at an early stage of MWM training. $\boldsymbol{a}$, Two cohorts of naive male room, and mice were released at the north or south point. The Day 2 mice failed to exhibit a preference for relative $(n=6)$ 列 pronounced in the mice that received the probe test after $4 \mathrm{~d}$ of training. $c$, The polar plot of the distribution of individual heading error measures (deviations from a direct path to the relative search zone) indicates that scores were clustered at $\sim 0^{\circ}$, indicating relative responding. The mean heading error for Day 2 mice probed after $2 \mathrm{~d}$ of training was $19.8^{\circ}$, indicated by the heading erro

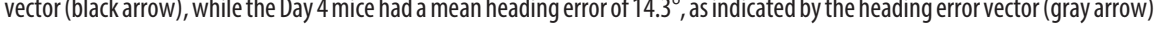
me release point and the location where the mice searched for the platform both shifted by $\sim 90^{\circ}$. The mice exhibited fore error scores shifted by $\sim 90^{\circ}$. The mean heading error was $-105.5^{\circ}$, as indicated by the heading error vector (arrow). These data indicate that the distal cues exert stimulus control over the platform search behavior of male $57 \mathrm{BL} / 6 \mathrm{~J}$ mice, suggesting that the mice engaged in platform search in the shifted absolute location. Error bars indicate SEM.

the pool $\left(F_{(2,79)}=1.15\right.$, n.s. $)$, or the duration spent in the relative search zone of the pool $\left(F_{(2,79)}=2.71\right.$, n.s. $)$. These results suggest that all mice exhibited a preference for relative responding in the water maze during the probe test, whether or not the pool was translated linearly in the room before the test. This preference for relative responding by male $\mathrm{C} 57 \mathrm{BL} / 6 \mathrm{~J}$ mice in the translated water maze is consistent with previous reports of equivalent responding by male Long-Evans rats in the water maze (Hamilton et al., 2007, 2008).

\section{Heading error}

To further quantify the relative responding of the mice, a heading error value was calculated for the initial portion of probe test swim path (Fig. $2 G$ ) for the mice of the relative versus absolute and the relative-only conditions. The portion of the path from the release point to the first entry into either the relative zone or the opposite zone was plotted and analyzed by determining the deviation in degrees of the actual path from a direct path to the relative zone (i.e., considered $0^{\circ}$ error). Since the analyses described earlier indicated no effect of pool position on training or 
a

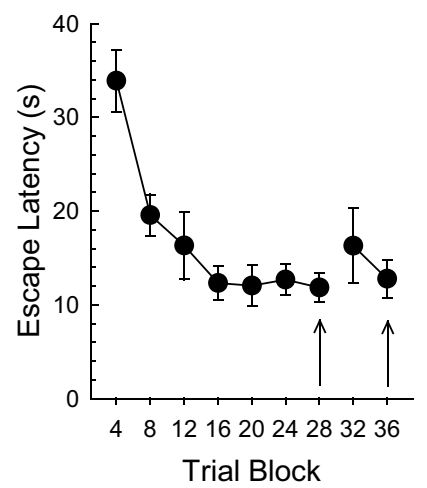

b

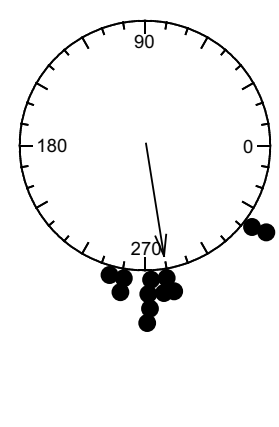

C

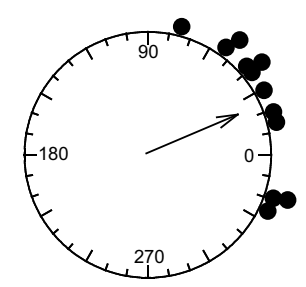

C57BL/6J mice exhibit a preference for relative over absolute responding even early in the course of Morris water maze training

It is possible that the marked preference of C57BL/6J mice for relative responding over absolute responding may be because the probe test in the translated pool was presented after the mice had acquired asymptotic performance in the MWM. In fact, mice may exhibit a transient preference for absolute responding early in training. To address this concern, distinct cohorts of 12 naive male C57BL/6J mice were trained in the MWM with the pool in the west position and the platform in position $\mathrm{B}$ for four trials per day for 2 or $4 \mathrm{~d}$. Analyses of the training data revealed a significant effect of training day on escape latency (Fig. $3 A$ ) and on the cumulative distance to platform center measures (both $F_{(3,33)}>12$, $p$ values $<0.001$ ). Just before the probe test, the pool was translated to the east position and the platform removed from the pool. For those mice probed after $2 \mathrm{~d}$ of training, there was no preference for one search strategy over another; six mice swam first to the relative zone, and the other six mice swam first to the absolute zone. Furthermore, there was no difference in latency to first enter the relative or absolute zone $\left(t_{(11)}=1.21\right.$, n.s.; Fig. $3 B$ ). Heading error measures for each mouse probed after $2 \mathrm{~d}$ of training (Fig. $3 C$ ) indicate a rather broad distribution of error scores (mean, $19.8 \pm 13.0^{\circ}$ ). Additional analyses of number of entries into, or dwell times in, the relative or absolute search zone also indicated a lack of significant search zone preference (both $t_{(11)}<$ 1, n.s.). These results suggest that at this early stage of training, there is no evidence for absolute or relative responding.

The cohort of mice probed after $4 \mathrm{~d}$ of training exhibited a preference for relative responding $(n=8)$ over absolute responding $(n=4)$. However, in light of the conservative estimate for the expected distribution of absolute and relative responses used in the $\chi^{2}$ analysis, this probe test results, the heading error data from mice probed in the east pool position and the west pool position were combined. The mean ( \pm circular SEM) heading error of the relative versus absolute condition was $10.6 \pm 6^{\circ}$, and the mean heading error of the relative-only condition was $10.1 \pm 5.6^{\circ}$. The distributions of heading error scores for both conditions are plotted in polar coordinates in Figure $2 \mathrm{~F}$. The polar plots reveal a predominant cluster of error scores from $0-10^{\circ}$, consistent with relative responding and a second, smaller cluster of error scores near $60^{\circ}$, consistent with absolute responding. Rayleigh test results revealed that the distribution of heading errors of each respective condition was significantly different from random (both $p$ values $<0.0001$ ).

preference for relative responding did not reach significance $\left(\chi^{2}=\right.$ 1.33 , n.s.). Although the mice had a shorter latency to first enter the relative zone (16.6 s) compared to the absolute zone ( $23.2 \mathrm{~s})$, this difference also did not reach significance $\left(t_{(11)}=1.95, p=\right.$ $0.07)$. The mean ( \pm circular SEM) heading error of the initial paths from release to arrival to the relative search zone (Fig. $3 C$ ) was $14.3 \pm 9.7^{\circ}$, a value that approximates that seen in mice trained in the task for $7 \mathrm{~d}$ (Fig. $2 F$ ). A Rayleigh test revealed that the distribution of heading errors was significantly different from random $(p<0.0001)$. These results suggest that a preference for relative responding over absolute responding develops progressively over the course of early MWM training in male C57BL/6J mice. This view is further supported by the fact that after $4 \mathrm{~d}$ of 
training, the mice made significantly more entries into the relative search zone than the absolute search zone $\left(t_{(11)}=-2.46, p=\right.$ 0.03 ), indicating concentrated search in the relative zone. These results are consistent with reports that rats exhibit a preference for relative responding during early stages of learning the MWM task (Hamilton et al., 2007, 2008). Furthermore, these results suggest that spatial search behavior of C57BL/6J mice does not progress from a preference for absolute responding to a preference for relative responding in the MWM.

\section{Extramaze cues exert stimulus control over platform search behavior in the nontranslated Morris water maze}

The predominance of relative responding by $\mathrm{C} 57 \mathrm{BL} / 6 \mathrm{~J}$ mice in the translated pool may reflect a stronger influence of intramaze apparatus cues rather than distal extramaze cues on spatial navigation in mice. If so, then platform search behavior of C57BL/6J mice should be insensitive to rotation of the distal visible cues. To determine whether platform search behavior is influenced by the extramaze cues, a naive cohort of mice were trained for only $4 \mathrm{~d}$ and then received a $30 \mathrm{~s}$ probe test in the nonshifted pool, with the extramaze cues rotated clockwise by $90^{\circ}$. Analyses of the training data revealed a significant effect of training day on escape latency (Fig. $3 C$ ) and on the cumulative distance to platform center measures (both $F_{(3,33)}>23, p$ values $<0.001$ ). Just before the $30 \mathrm{~s}$ probe test, the curtain, containing the extramaze visible cues, was rotated $90^{\circ}$ clockwise. The pool remained in the west position in the room. During the probe test, a majority of the mice $(n=9)$ swam first to the location in the pool corresponding to that predicted by the shifted extramaze cues (i.e., $\mathrm{A}^{\prime}$ zone); that is, these mice shifted their search responding $90^{\circ}$ clockwise, similar to the rotation of the visual cues. The remaining three mice swam first to the shifted opposite $\left(\mathrm{O}^{\prime}\right)$ zone. The latency to enter the $\mathrm{A}^{\prime}$ zone $(4.45 \mathrm{~s})$ was significantly shorter than that to enter the $\mathrm{O}^{\prime}$ zone $\left(15.40 \mathrm{~s} ; t_{(11)}=-3.09, p<0.015\right)$. The distribution of heading error scores is plotted in polar coordinates in Figure $3 D$. The mean ( \pm circular SEM) heading error of the initial paths from release to arrival at a search zone was $-105.5 \pm 30.2^{\circ}$. The Rayleigh test revealed that the distribution of heading errors was significantly different from random $(p<0.0001)$. These results clearly indicate that spatial search behavior of male C57BL/6J mice in the MWM is influenced by room-based or extramaze cues. This condition represented a cue conflict (i.e., rotated distal cues, but stable local or apparatus cues), and perhaps in such an instance the room-based reference frame guides behavior.

\section{Extramaze cues exert stimulus control over relative responding in the Morris water maze}

To determine whether the relative responding of the mice in the translated pool is guided by the exteroceptive cues, a probe test was conducted in the translated pool after $90^{\circ}$ rotation of the extramaze cues. Twelve naive male C57BL/6J mice were trained as above in the MWM over $7 \mathrm{~d}$ (four trials per day) to find the platform in position B in the pool positioned in the west of the testing room. Analyses of the training data revealed a significant effect of trial block on escape latency and on the cumulative distance to target measures (both $F_{(6,66)}>10$, $p$ values $<0.001$; Fig. $4 A$ ). Immediately following the last training trial, the pool was translated linearly in the testing room from the west to the east position. All of the mice in this experiment were tested as described above for the relative versus absolute probe condition. Just before the $30 \mathrm{~s}$ probe test, the extramaze cues on the curtain were rotated $90^{\circ}$ clockwise. Thus, this experiment was conducted to determine whether the relative responding of the mice in the translated pool is influenced by the extramaze or room-based cues. The possible release points were also rotated by $90^{\circ}$ so that mice were released into the pool at either the west or east point along the pool perimeter (Fig. 1C), and mice were permitted to swim for $30 \mathrm{~s}$.

Consistent with the preferential relative responding observed during the probe tests of the initial experiment, the mice first entered the $\mathrm{R}^{\prime}$ search zone, as opposed to the $\mathrm{A}^{\prime}$ search zone, consistent with their locations as dictated by the $90^{\circ}$ rotation of the extramaze cues (Fig. $4 C$, examples of initial swim paths). Analyses revealed an overwhelming and significant preference for the mice to exhibit relative responding $(n=10)$ as opposed to absolute responding $(n=2)$ during the probe test with the cues rotated $90^{\circ}\left(\chi^{2}=5.33, p<0.05\right)$. The latency of the mice to enter the $\mathrm{R}^{\prime}$ search zone $(5.23 \mathrm{~s})$ was significantly shorter than that to enter the $\mathrm{A}^{\prime}$ search zone $\left(15.84 \mathrm{~s} ; t_{(11)}=-2.94, p<0.015\right)$. Furthermore, the mean latency of these mice to enter the $\mathrm{R}^{\prime}$ search zone was similar to the mean latency of the relative versus absolute mice as depicted in Figure 2. The distribution of heading error scores are plotted in polar coordinates in Figure $4 \mathrm{~B}$. The mean ( \pm circular SEM) heading error of the initial paths from release to arrival at a search zone was $-81.6 \pm 6.9^{\circ}$. The Rayleigh test revealed that the distribution of heading errors was significantly different from random $(p<0.0001)$. Together, these data indicate that the rotation of the extramaze cues by $-90^{\circ}$ caused a near corresponding shift in relative responding of the mice during the probe test.

\section{Disorientation disrupts relative responding in the Morris water maze}

It was noted during the previous experiments that as the mice were being lowered into and then released into the pool, they would often adjust their orientation to align themselves in the direction of one of the search zones. Once released, the mice would swim in a direct and often ballistic path to the preferred search zone. This pattern of behavior suggests that the relative responding may also be guided by internally generated cues and the relationship of the entry point of the curtain with the release points at the pool edge. Therefore, this experiment examined the degree to which interoceptive cues (e.g., vestibular), and the mouse's view of cues, during the transit from the holding cage through the curtained area and into the pool, influence relative responding in the water maze. Twenty-four hours after the cue rotation probe test, the extramaze cues were returned to their original positions, and the 12 mice were retrained in the pool in the west position for four trials, and for another four trials on the following day. Performance of the mice on these retraining trials was consistent with that of the preceding trials (Fig. $4 A$, compare trial blocks 24-28, 32-36).

After the 36th training trial, the pool was translated linearly in the testing room from the west to the east position. Each mouse was disoriented before the probe test by placement into a closed corrugated cardboard box and gently rotated clockwise and counterclockwise for $60 \mathrm{~s}$ as the experimenter walked around the pool. Afterward, the closed box was placed on the lab bench for $30 \mathrm{~s}$ and then carried through the curtain area to the side of the pool at the west or east release point. The box was opened and the mouse placed gently into the water maze for the $30 \mathrm{~s}$ probe test. All other probe test procedures were exactly as described for the relative versus absolute condition above. Upon release into the pool, the mice tended to swim in a circuitous path before swimming toward the relative or absolute search zone. The disorientation procedure appeared to impair the preference for relative 
responding, as there was a lack of significant preference for choosing the relative $(n=7)$ over the absolute search zone $(n=$ 5 ) during the initial swim path of the probe test $\left(\chi^{2}=0.33\right.$, n.s.). There was also no significant difference in the latency to first enter the relative or absolute search zones $\left(t_{(11)}=0.08\right.$, n.s. $)$. The distribution of heading error measures for each mouse in the disorientation probe trials is plotted in polar coordinates in Figure $4 D$. The mean ( \pm circular SEM) heading error of the initial paths from release to arrival at a search zone was $25.4 \pm 10.2^{\circ}$. Despite the finding that heading error measures of disoriented mice were more distributed than those of Figure $2 F$, a Rayleigh test revealed that the distribution of heading errors was significantly different from random $(p<0.0001)$. These results reveal that disorientation impaired the preference of the mice to exhibit relative responding in the water maze. Furthermore, the representative swim paths (Fig. $4 E$ ) reveal that during this probe test, the mice often swam in a looping manner, making turns of $360^{\circ}$ or so, before continuing on, suggesting that the mice were disoriented with respect to platform location. This result suggests that under the normal conditions, the information that supports relative responding in mice includes vestibular cues. This conclusion is supported by prior studies in which vestibular cues are critical for spatial learning and navigation in rodents (Potegal et al., 1977; Semenov and Bures, 1989; Stackman and Herbert, 2002; Wallace et al., 2002; DeCoteau et al., 2004).

\section{Inactivation of the anterior thalamus abolishes relative responding of $\mathrm{C} 57 \mathrm{BL} / 6 \mathrm{~J}$ mice in the Morris water maze} Histological analyses confirmed accurate placement of microinfusion sites within the anterodorsal thalamic nuclei of 14 mice (Fig. 5A,B). In some cases, the track of the infusion cannulae extended to the ventral extent of the anterodorsal thalamic nuclei, encroaching on the anteroventral and anteromedial thalamic nuclei. There were no significant differences between behavioral performance for mice in which muscimol may have affected the anteroventral thalamic nuclei compared to those with cannulae located more dorsally. Given the estimated spread of microinfused muscimol with the tissue, we interpret these results as the consequence of inactivation of the anterodorsal and anteroventral thalamic nuclei, or the ATN. Following implantation of chronic bilateral guide cannulae, C57BL/6J mice were trained as described previously over $7 \mathrm{~d}$ (four trials per day) to find the platform in position A or B in the pool positioned in the west of the testing room. There was a main effect of trial block on both escape latency $\left(F_{(6,78)}=15.65, p<0.001\right.$; Fig. $\left.6 A\right)$ and the cumulative distance to target measures $\left(F_{(6,78)}=12.45, p<\right.$ $0.001)$. Each mouse received an intra-anterior thalamic microinfusion after the 28th training trial and then a 30 s probe test in the platformless pool after it was translated linearly to the east position in the testing room. Twenty-four hours after the first intrathalamic infusion, mice were retrained for four trials in the water maze in the west position. Mice received four more training trials on the following day before receiving the second intra-anterior thalamic microinfusion. During each intrathalamic microinfusion session, half of the cohort received aCSF, and the remaining mice received muscimol. On the subsequent microinfusion session, the treatment assignments were reversed to achieve a within-subjects crossover design. Escape latency measures for the 2 preinfusion days were averaged and compared to the average for the 2 postinfusion days using a paired-samples $t$ test $\left(t_{(13)}=-0.03\right.$, n.s. $)$. The same analysis was used to compare cumulative distance to platform center (CDT) values for preinfusion and postinfusion training
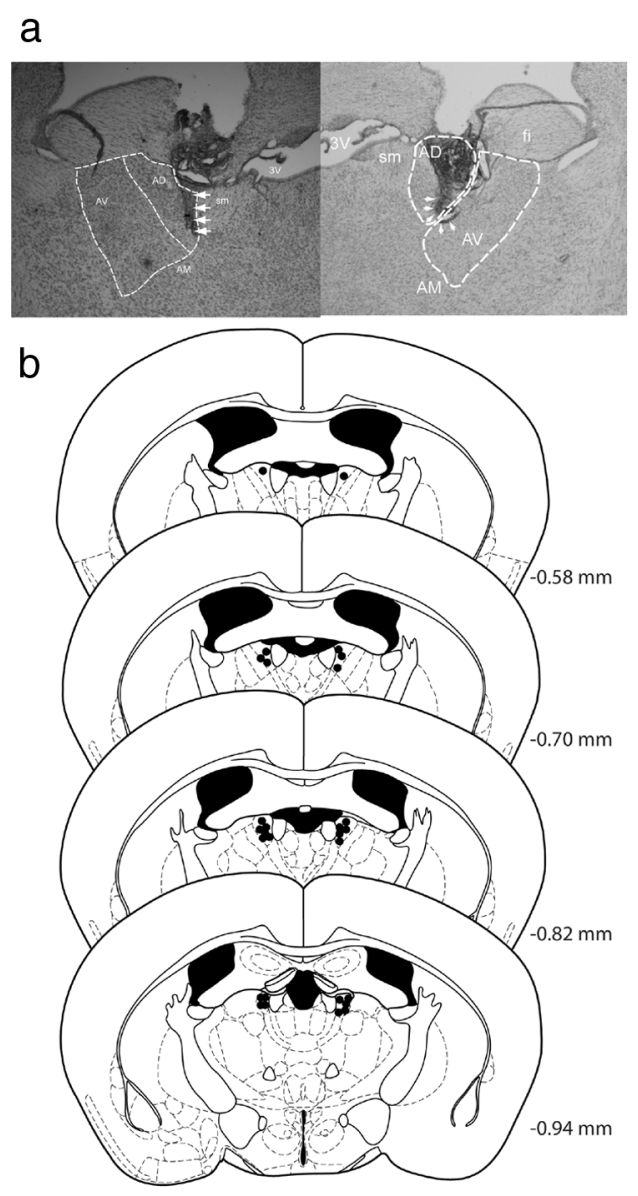

Figure 5. Histological verification of bilateral infusion cannulae placement into the anterior thalamic nuclei. $\boldsymbol{a}$, Representative photomicrographs depicting infusion cannula track through the ATN in the left hemisphere (left) and right hemisphere (right). The anterodorsal thalamic nuclei (AD), anteroventral thalamic nuclei (AV), anteromedial thalamic nuclei (AM), fimbria (fi), stria medullaris (sm), and the third ventricle (3V) are outlined or identified in white. Arrowheads indicate the track of the infusion cannula. $\boldsymbol{b}$, The individual placements of infusion sites (filled black circles) for the 14 mice are presented against the atlas plates from -0.58 to -0.94 $\mathrm{mm}$ from bregma (Franklin and Paxinos, 2008).

$\left(t_{(13)}=-0.49\right.$, n.s. $)$. These results indicate that the intrathalamic microinfusions had no lasting effects on the ability of the mice to navigate to the platform.

Mice were assigned to either the relative versus absolute or relative-only probe test condition, and run accordingly as described previously. There were no differences in relative responding between these two conditions, and therefore all data were combined into one group for analysis. After receiving aCSF microinfused into the ATN, the mice exhibited a preference for relative responding over absolute responding $\left(\chi^{2}=10.28, p<\right.$ 0.001 ; Fig. $6 B$ ), consistent with the results shown in Figure 2. In contrast, after bilateral microinfusion of muscimol into the ATN, the same mice failed to exhibit relative responding, and instead exhibited a preference for absolute responding $\left(\chi^{2}=4.86, p<\right.$ 0.01 ; Fig. $6 B$ ). After receiving intrathalamic muscimol, two of the mice failed to choose either the relative or absolute/opposite search zone during the $30 \mathrm{~s}$ probe test. These two mice swam in a tight circling pattern and remained close to the release point throughout the test. However, both mice exhibited typical relative responding after intra-ATN aCSF. The data for these two mice were eliminated from the subsequent analyses due to the lack of a matching set of data for both treatment conditions. 
a

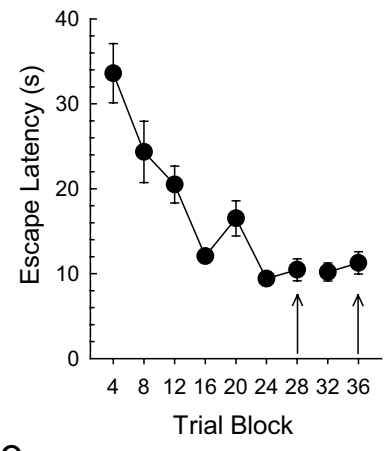

b

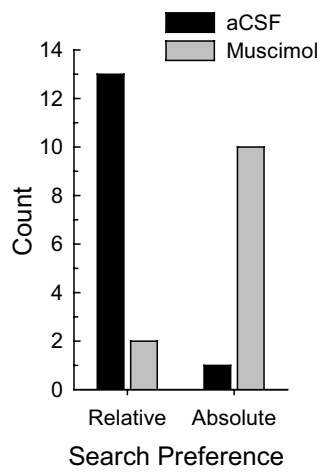

C

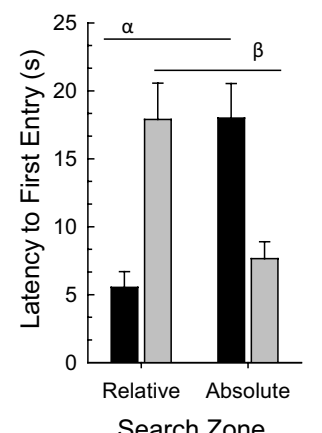

d

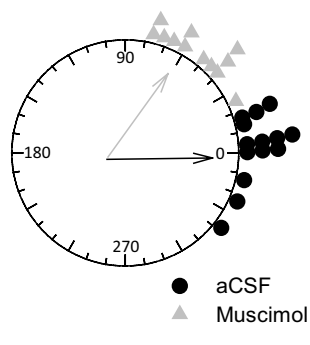

e

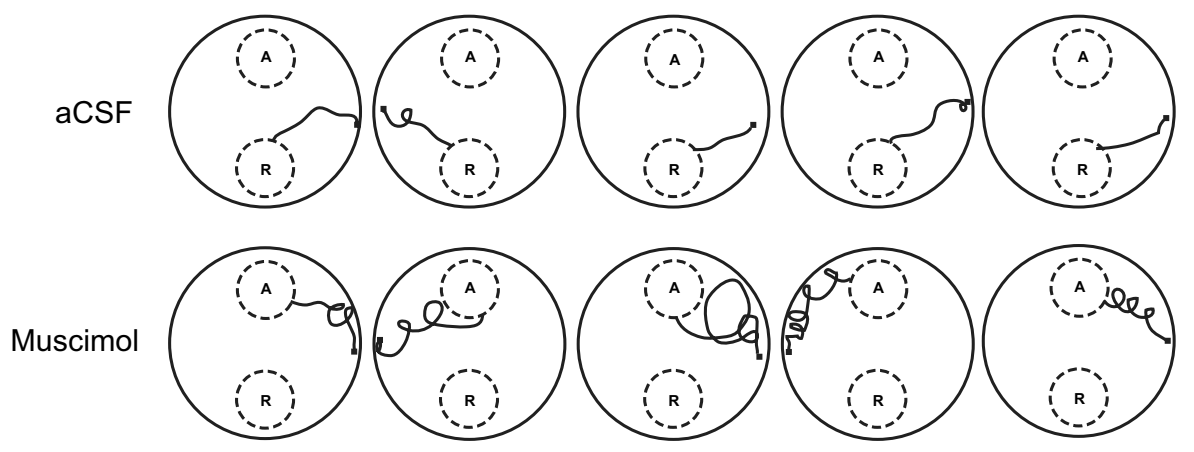

Figure 6. Inactivation of the anterior thalamic nuclei abolishes relative responding in the water maze. $a$, A naive cohort of 14 male C57BL/6J mice were trained for 28 trials in the water maze in the west position of the testing room. Each mouse received a bilateral microinfusion of aCSF or muscimol ( $2 \mu \mathrm{g} / 0.25 \mu \mathrm{l} / \mathrm{side}) 20 \mathrm{~min}$ before the probe test in the translated pool. Mice were trained for eight more trials over the following $2 \mathrm{~d}$ and then received a second bilateral microinfusion of aCSF or muscimol with treatment assignments reversed from those of the first microinfusion. Twenty minutes after each microinfusion, each mouse received a 30 s probe test (arrows) in the translated pool. $\boldsymbol{b}$, The mice exhibited a strong preference for relative responding over absolute responding after intra-anterodorsal thalamic aCSF, consistent with results shown in Figure 2 . In contrast, the mice exhibited an overwhelmingly preference for absolute responding over relative responding after intra-anterodorsal thalamic muscimol. c, After aCSF microinfusion, the mice exhibited significantly shorter latencies to the relative search zone than the absolute search zone ( $\left.{ }^{\alpha} p<0.001\right)$. After muscimol microinfusion, the mice instead exhibited significantly shorter latencies to the absolute search zone than the relative search zone ( ${ }^{\beta} p<0.001$ ). $\boldsymbol{d}$, The polar plot of the distribution of individual heading error scores for the mice reveal that scores for aCSF treatment were clustered at $\sim 0^{\circ}$ (mean heading error, $1.2^{\circ}$, black arrow; length of the heading error vector, $r=0.96$ ), while those of muscimol treatment were clustered at $\sim 60^{\circ}$ (mean, $54.8^{\circ}$, gray arrow; length of the heading error vector, $r=0.96$ ), consistent with absolute responding. $e$, Representative swim paths for five of the mice indicate that after intra-anterodorsal thalamic aCSF (top), swim paths were direct to the relative search zone, while after intra-anterodorsal thalamic muscimol, the same mice (bottom) swam to the absolute search zone via a much less direct and more circuitous paths. Error bars indicate SEM.

The latency to first enter the relative or absolute search zone was analyzed by a two-factor (treatment and search zone) repeated-measures ANOVA. The ANOVA yielded only a significant treatment by search zone interaction $\left(F_{(1,11)}=40.58\right.$, $p<0.0001$; Fig. 6C). The lack of a significant main effect of treatment or search zone is due to the completeness of the interaction: the infusion of muscimol into the ATN fully reversed the preference of the mice for relative responding over absolute responding. Mice receiving aCSF consistently searched for the platform in the relative direction (i.e., relative search zone), while the intrathalamic muscimol mice consistently searched in the location where the platform had been during training, as dictated by the extramaze cues (i.e., absolute search zone). Representative initial swim paths are presented in Figure 6E, illustrating the pattern of responding observed for five mice after intrathalamic aCSF (top) and that for the same mice after intrathalamic muscimol (bottom). While aCSF-treated mice executed direct paths to the respective search zone (i.e., R), the paths of the muscimol-treated mice were less direct and comprised of longer spiraling searches, with most mice arriving in the A search zone. These results indicate that inactivation of the ATN impaired relative responding in mice that preferred this behavioral strategy to absolute responding when the ATN were functionally intact.

Heading error measures were calculated for each probe test under each respective intrathalamic condition. These data, plot- ted in Figure $6 D$, further confirm the ATN inactivation-induced switch in search preference. The mean heading error for the mice when treated with intrathalamic aCSF was $1.2 \pm 4.6^{\circ}$, which is indicative of relative responding. In contrast, when treated with intrathalamic muscimol, the mean heading error of the mice was $54.8 \pm 5.1^{\circ}$, corresponding to absolute responding. A pairwise Watson-Williams $F$ test, used to analyze the effect of treatment on angles of heading error, yielded a significant effect of treatment $\left(F_{(1,36)}=9.3, p<0.005\right)$. Together with those above, these results suggest that the relative responding of mice in the water maze is dependent on the functional integrity of the anterior thalamic nuclei.

\section{Relative responding is intact after inactivation of the dorsal CA1 of C57BL/6J mice in the Morris water maze}

Histological analyses confirmed accurate placement of bilateral microinfusion sites within the dorsal CA1 region of the hippocampus of $11 \mathrm{C} 57 \mathrm{BL} / 6 \mathrm{~J}$ mice (Fig. $7 A, B$ ). Following implantation of chronic bilateral guide cannulae, the mice were trained over $7 \mathrm{~d}$ (four trials per day) to find the platform in position A or $\mathrm{B}$ in the pool positioned in the west of the testing room. There was a main effect of trial block on both escape latency $\left(F_{(6,60)}=13.07\right.$, $p<0.001$; Fig. $8 A)$ and on the cumulative distance to target measures $\left(F_{(6,60)}=13.12, p<0.001\right)$. Each mouse received a bilateral intrahippocampal microinfusion after the 28th training trial and then a $30 \mathrm{~s}$ probe test in the platformless pool after it was 

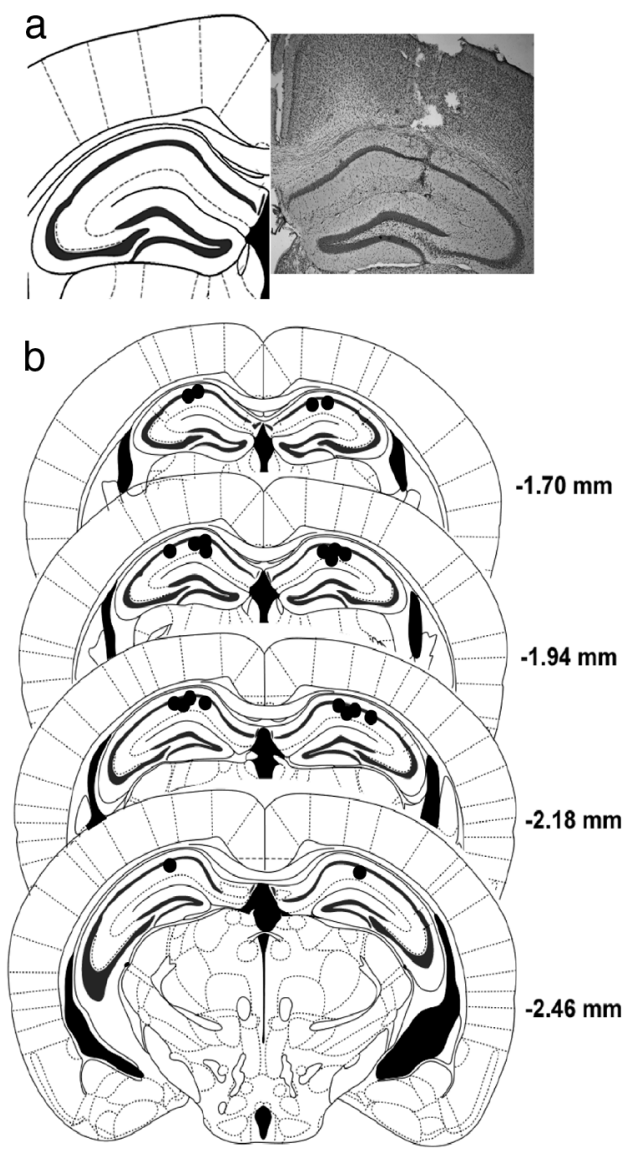

Figure 7. Histological verification of bilateral infusion cannulae placement into the CA1 region of the dorsal hippocampus. $\boldsymbol{a}$, A representative photomicrograph depicting the infusion cannula track through the right dorsal CA1 region (right). The left panel depicts the left dorsal CA1 region for comparison purposes, from the mouse brain atlas of Franklin and Paxinos (2008). $\boldsymbol{b}$, The individual placements of infusion sites (black symbols) for the 11 mice are presented against the atlas plates from -1.70 to $-2.46 \mathrm{~mm}$ from bregma.

translated linearly to the east position in the testing room. Twenty-four hours after the first intrahippocampal microinfusion, mice were retrained for four trials in the water maze in the west position. Mice received four more training trials on the following day before receiving the second intrahippocampal microinfusion. During each intrahippocampal microinfusion session, half of the cohort received aCSF, and the remaining mice received muscimol. On the subsequent microinfusion session, the treatment assignments were reversed to achieve a within-subjects crossover design. Escape latency measures for the 2 preinfusion days were averaged and compared to the average for the 2 postinfusion days using a paired-samples $t$ test $\left(t_{(10)}=0.04\right.$, n.s. $)$. The same analysis was used to compare CDT values for preinfusion and postinfusion training $\left(t_{(10)}=-0.70\right.$, n.s.). These results indicate that the intrahippocampal microinfusions had no lasting effects on the ability of the mice to navigate to the platform.

Mice were assigned to the relative versus absolute or relative only probe test condition and run accordingly. As observed in previous experiments, there were no differences in relative responding between these two conditions, and therefore all data were combined into one group for analysis. Interestingly, all mice, regardless of bilateral intrahippocampal microinfusion of aCSF or muscimol, exhibited a preference for relative responding over absolute responding (both $\chi^{2}=4.55, p<0.04$; Fig. $8 B$ ), consistent with the results shown in Figure 2. Although the total numbers of mice choosing relative responding compared to absolute responding are identical across the two treatment conditions, there were some differences in search preference for individual mice according to treatment. Under the influence of intrahippocampal aCSF, two mice exhibited absolute responding, but these same mice exhibited relative responding when tested after intrahippocampal muscimol. Two other mice that exhibited relative responding after intrahippocampal aCSF then exhibited absolute responding after intrahippocampal muscimol. Thus, there was no clear pattern of effect of intrahippocampal microinfusion on platform search behavior.

The latency to first enter the relative or absolute/opposite search zone was analyzed by a two-factor (treatment and search zone) repeated-measures ANOVA. The ANOVA yielded only a significant main effect of search zone $\left(F_{(1,22)}=23.57, p<0.0001\right.$; Fig. $8 C)$. The lack of a significant main effect of treatment or a treatment by search zone interaction indicates that the preference of the mice for relative responding over absolute responding was not influenced by the intrahippocampal treatment. Representative initial swim paths are presented in Figure $8 E$, illustrating the pattern of responding observed for five mice after intrahippocampal aCSF (top) and that for the same mice after intrahippocampal muscimol (bottom). As observed after intrathalamic microinfusions, the aCSF-treated mice swam in direct paths to the respective search zone. After intrahippocampal microinfusion of muscimol, the mice swam in paths that were less direct, and these mice preferred to swim to the relative search zone. These results indicate that inactivation of the dorsal CA1 region of the hippocampus did not interfere with relative responding in mice during the probe test in the translated water maze.

Heading error measures were calculated for each probe test under each respective intrahippocampal condition. These data, plotted in Figure $8 D$, further confirm the consistency of relative responding across the two intrahippocampal treatment conditions. The mean heading error for the mice when treated with intrahippocampal aCSF was $10.4 \pm 9.4^{\circ}$, and when treated with intrahippocampal muscimol, the mean heading error of the mice was $11.4 \pm 10.3^{\circ}$, both of which are indicative of relative responding. A pairwise Watson-Williams $F$ test, used to analyze the effect of intrahippocampal treatment on angles of heading error, yielded a nonsignificant effect of treatment $\left(F_{(1,20)}=0.007\right.$, n.s. $)$. Collectively, these results suggest that the relative responding of mice in the water maze is not dependent on the functional integrity of the dorsal hippocampus. Previous reports indicate that permanent lesions of the hippocampus impair directional learning in the T-maze (Stringer et al., 2005) and impair postsurgical performance of a working memory task that required rats to maintain direction information (DeCoteau et al., 2004). Our result that hippocampal inactivation spares relative responding would appear to conflict with these prior reports. It is difficult to align behavioral effects of permanent and temporary lesions due to the possible postlesion compensatory changes in the brain. However, hippocampal lesions do not disrupt HD cell activity in ATN or postsubiculum (Golob and Taube, 1997), so it is possible that the intact relative responding after temporary hippocampal inactivation is guided by the ATN.

\section{Infusion of FCM into the anterodorsal thalamic nuclei impairs relative responding, while infusion of FCM into the dorsal CA1 spares relative responding}

The effect on relative responding of FCM microinfusion into the anterodorsal thalamic nuclei or the dorsal CA1 region of the hippocampus was also assessed. This examination was conducted 
a

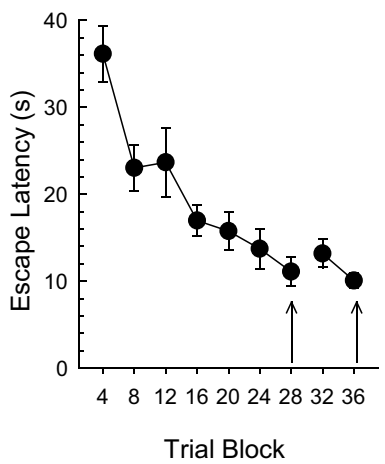

b

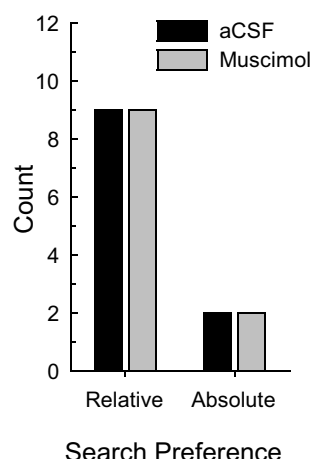

c

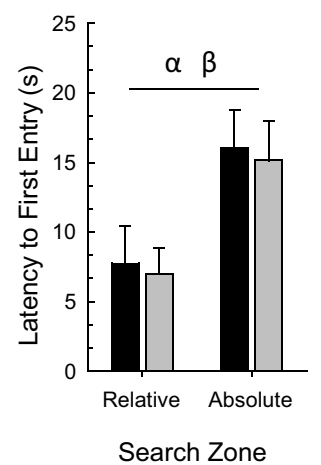

d

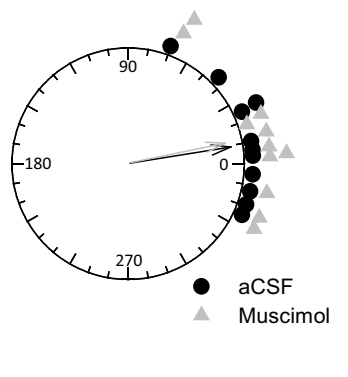

e
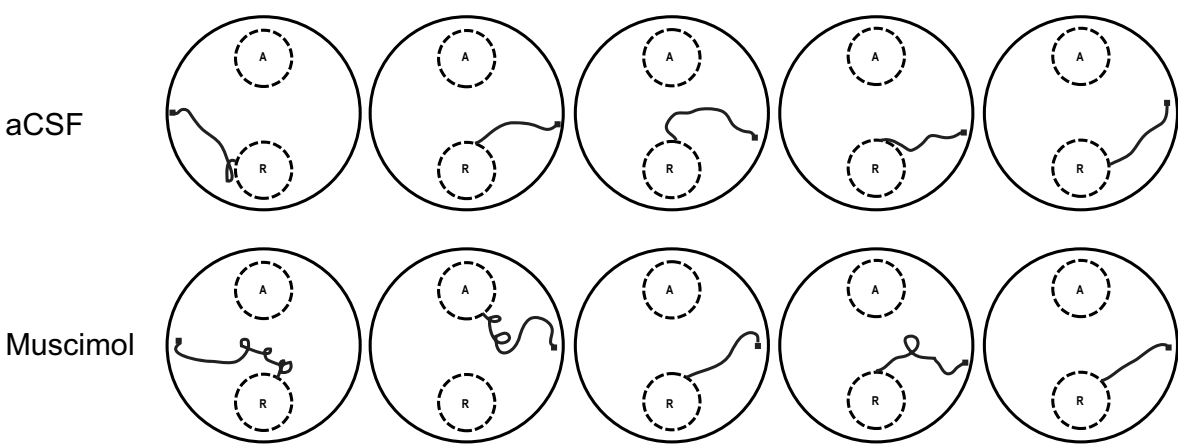

Figure 8. Relative responding is not affected by inactivation of the dorsal CA1 region of the hippocampus. This experiment was conducted identically to that presented in Figure 6, except that mice received bilateral microinfusion of muscimol or aCSF into the CA1 region of dorsal hippocampus before a 30 s probe test in the translated pool. $\boldsymbol{a}$, Eleven naive male $(57 B L / 6$ J mice were trained for 28 trials in the water maze in the west position of the testing room. Twenty minutes after microinfusion of aCSF or muscimol ( $2 \mu \mathrm{g} / 0.25 \mu \mathrm{l} / \mathrm{side})$, each mouse received a $30 \mathrm{~s} \mathrm{probe} \mathrm{test} \mathrm{in} \mathrm{the}$ translated pool (arrows). $\boldsymbol{b}$, The mice exhibited a strong preference for relative responding over absolute responding after intra-CA1 aCSF, consistent with results shown in Figure 2 . Interestingly, relative responding was also observed after intra-CA1 muscimol. $c$, After intra-CA1 aCSF or muscimol, mice exhibited significantly shorter latencies to the relative search zone than the absolute search zone $\left({ }^{\alpha, \beta} p<0.001\right.$ ). $\boldsymbol{d}$, The polar plot of the distribution of individual heading error scores for the mice shows clustering at $\sim 10^{\circ}$ (aCSF mean heading error vector, $10.4^{\circ}$, black arrow, $r=0.89$; muscimol mean heading error vector, $11.4^{\circ}$, gray arrow, $r=0.87$ ), indicative of relative responding. $e$, Representative swim paths for five of the mice indicate that after intra-CA 1 infusion of aCSF (top) or muscimol (bottom), swim paths were direct to the relative search zone. Error bars indicate SEM.

to better estimate the degree of spread of microinfused muscimol within the respective target tissue. Naive male C57BL/6J mice were implanted with chronic bilateral guide cannulae as described previously. Upon postoperative recovery, the mice were trained to the platform in location B in the Morris water maze task with the pool in the west position of the testing room. Upon acquiring the task, each mouse received bilateral microinfusion of FCM or PBS vehicle into the anterodorsal thalamic nuclei or dorsal CA1 region of the hippocampus. Ten minutes after the completion of the microinfusion, each mouse received a $30 \mathrm{~s}$ probe test with the pool translated to the east position of the testing room. On the following $2 \mathrm{~d}$, all mice received four more training trials, and then a second microinfusion and another $30 \mathrm{~s}$ probe test. The treatment assignments were reversed for the second probe test. All mice received a second FCM microinfusion and were perfused, and then brains were sectioned at $50 \mu \mathrm{m}$ and were analyzed to determine the spread of FCM. Results described below are for all mice determined to have appropriate cannulae placements.

Histology revealed cannulae placements in the anterodorsal thalamic nuclei or the dorsal CA1 region of the hippocampus. Visualization of FCM microinfusion in $50 \mu \mathrm{m}$ coronal tissue sections showed that after microinfusion into the anterodorsal thalamic nuclei, the fluorescence was restricted to the anterodorsal thalamic nuclei, with some spread in the lateral and ventral directions to the dorsomedial anterior ventral thalamic nuclei, ventrolateral anterior ventral thalamic nuclei, and anteromedial thalamic nuclei, and minimal spread in the medial direction to the stria medullaris of thalamus (Fig. 9A,B). Estimates of the anterior-posterior spread of FCM from visualization of sequential $50 \mu \mathrm{m}$ tissue sections indicated that the FCM was confined to within $\pm 250-300 \mu \mathrm{m}$ of the infusion cannulae tracks. In two mice, weak fluorescence was also detected dorsal to the anterodorsal thalamic nuclei surrounding the ventralmost tips of the guide cannulae. Given the proximity of the anterodorsal thalamic nuclei to the lateral ventricle, it is conceivable that FCM may have diffused dorsal along the tracks of the microinfusion cannulae. Visualization of FCM microinfusion into the dorsal CA1 region of the hippocampus revealed that fluorescence was restricted to the area surrounding the pyramidal cell layer but spanning the strata oriens, radiatum, and lacunosum moleculare (Fig. 9D,E). The anterior-posterior spread of the FCM within the hippocampal appeared to span approximately $\pm 300 \mu \mathrm{m}$. It is possible that intrahippocampal FCM remained restricted to the hippocampus given that white matter fibers may have acted as diffusion barriers, consistent with a previous report (Allen et al., 2008).

Following PBS microinfusions into the anterodorsal thalamic nuclei, mice exhibited a preference for relative responding ( ix of seven mice). In contrast, mice that received FCM microinfusion into the anterodorsal thalamic nuclei failed to exhibit relative responding and instead first entered the absolute search zone (seven of seven mice). A two-factor (treatment and search zone) repeated-measures ANOVA on 
a

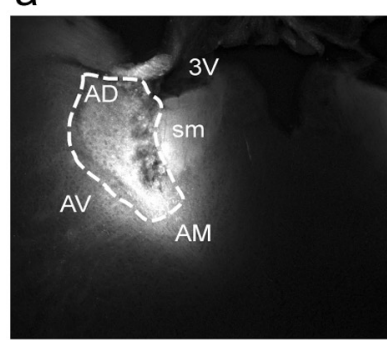

b

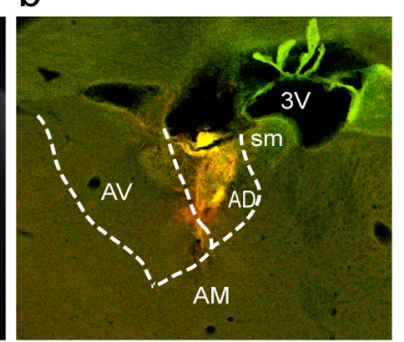

d

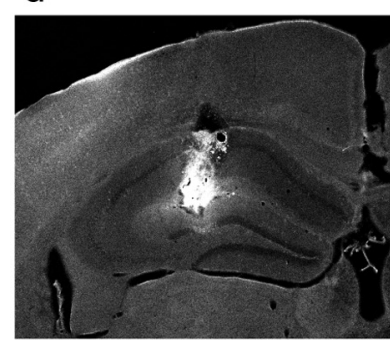

e

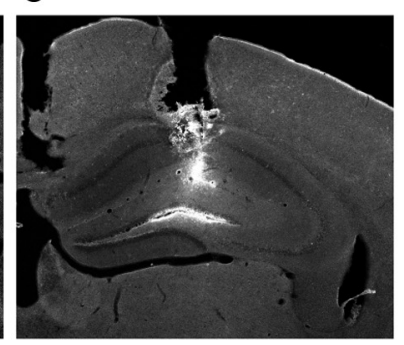

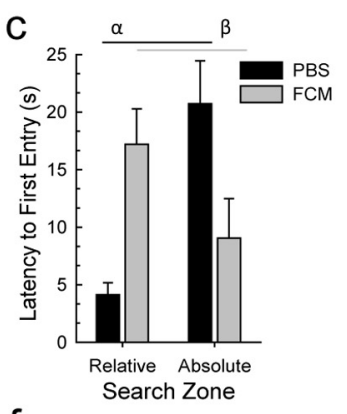

$f$

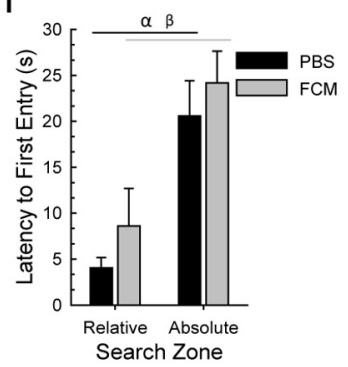

Figure 9. Relative responding is impaired after intra-anterior thalamic FCM, but spared after intra-CA1 FCM. Naive C57BL/6J mice $(n=9)$ were implanted with bilateral cannulae directed at the anterodorsal thalamic nuclei. The mice were then trained for 28 trials in the water maze in the west position to find the platform at position B, as described for the other experiments. Twenty minutes before a 30 s probe test in the translated pool, the mice received bilateral microinfusion of FCM or PBS vehicle. After $2 \mathrm{~d}$ of retraining in the west, the mice received a second microinfusion when treatment assignments were reversed. $\boldsymbol{a}$, Epifluorescent image acquired at $4 \times$ magnification illustrating the distribution of FCM within the left ATN from a representative $50 \mu \mathrm{m}$ coronal section. The fluorophore can be seen within the anterodorsal nuclei (AD; outlined with dashed white line), but also appears to have spread into the anteroventral nuclei (AV) and anteromedial nuclei (AM) as well. 3V, Third ventricle; sm, stria medullaris. $\boldsymbol{b}$, Epifluorescent image illustrating the distribution of FCM (red) after intrathalamic microinfusion against green fluorescent background staining for Nissl (NeuroTrace; Invitrogen). The dashed white line depicts the boundary of the anterodorsal thalamic nuclei. c, Microinfusion of FCM into the ATN abolished relative responding observed after intrathalamic PBS. Intrathalamic PBS-treated mice swam first to the relative search zone during the probe test in the translated pool ( $\left.{ }^{\alpha} p<0.01\right)$. In contrast, intrathalamic FCM-treated mice swam first to the absolute search zone, consistent with absolute responding $\left({ }^{\beta} p<0.01\right)$. $\boldsymbol{d}, \boldsymbol{e}$, Epifluorescent images from two cases acquired at $4 \times$ magnification, illustrating the distribution of FCM within representative $50 \mu \mathrm{m}$ coronal sections through the dorsal hippocampus after bilateral microinfusion into the CA1 region. The fluorophore appears to be distributed within the dorsal hippocampus with some spread ventrally to the dentate gyrus. $f$, Microinfusion of FCM into the dorsal CA1 did not affect relative responding of mice. Intra-CA1 PBS-treated mice swam first to the relative search zone, as did the same mice after intra-CA1 FCM ( $\left.{ }^{\alpha} p<0.01 ;{ }^{\beta} p<0.04\right)$. Error bars indicate SEM.

measures of latency to first enter the relative or absolute search zone yielded only a significant treatment by search zone interaction $\left(F_{(1,6)}=39.38, p<0.002\right.$; Fig. $\left.9 C\right)$ for the anterodorsal thalamic nuclei group.

Following PBS microinfusions into the dorsal CA1 region of the hippocampus, mice exhibited a preference for relative responding (seven of nine mice). Mice that received FCM microinfusion into the dorsal CA1 also exhibited relative responding (seven of nine mice; one mouse that failed to enter either the relative or absolute zone in $30 \mathrm{~s}$ ). A two-factor (treatment and search zone) repeated-measures ANOVA on measures of latency to first enter the relative or absolute search zone yielded a significant effect only of search zone $\left(F_{(1,8)}=13.95, p<0.005\right.$; Fig. $\left.9 F\right)$ for the dorsal CA1 group.

The behavioral performance of mice after microinfusion of FCM into the anterodorsal thalamic nuclei or the dorsal CA1 region of the hippocampus was equivalent to that observed in mice after microinfusion of muscimol into these respective regions. In light of the observed spread of the FCM, the results of intra-anterodorsal thalamic nuclei infusions should be interpreted as the influence of inactivation of the ATN. Furthermore, it should be noted that the distribution of microinfused FCM might not have duplicated the precise extent of spread of micro- infused muscimol, since the fluorophore tag may have influenced the distribution of FCM. Therefore, it is difficult to infer definitively the degree to which muscimol diffuses beyond the borders of the region of interest.

\section{Discussion}

Linear translation of the MWM from its position during training to that during a probe test revealed that rather than swimming to the absolute trained location (i.e., absolute responding), rats prefer to swim in the same direction from the release point that would take them to the platform during training (i.e., relative responding) (Hamilton et al., 2008). Here, male C57BL/6J mice were found to exhibit an overwhelming preference for relative responding over absolute responding during a probe test imposed either early or late in MWM training, results that are consistent with similar findings of rats (Hamilton et al., 2007, 2008, 2009). In the current study, a $90^{\circ}$ rotation of extramaze cues caused a corresponding shift in relative responding, indicating that distal visual cues exert stimulus control over the relative responding of the mice. Furthermore, disorientation by gentle rotational stimulation disrupted relative responding. Disorientation may have impaired the animals' use of interoceptive cues, and the longer latencies to enter the absolute or relative search zone may reflect the need for disoriented mice to assess exteroceptive cues before planning trajectories to the remembered platform location. Such manipulations of exteroceptive and interoceptive cues affected relative responding in much the same way as these manipulations affect HD cells recorded from the ATN (Taube, 1995, 2007). Temporary bilateral muscimol- or FCM-induced inactivation of the ATN abolished relative responding of $\mathrm{C} 57 \mathrm{BL} / 6 \mathrm{~J}$ mice in the MWM by essentially reversing the search preference of the mice from relative responding to absolute responding. During probe tests, mice that received intrathalamic muscimol swam in paths that were considerably less direct, further demonstrating the disruption of relative responding. These findings suggest that the intrathalamic muscimol-treated mice were disoriented; yet over the course of the probe trial, these mice did tend to arrive at the previous platform location, perhaps after referencing the extramaze cues. Together, these results suggest that the predominant response strategy used by $\mathrm{C} 57 \mathrm{BL} / 6 \mathrm{~J}$ mice in the water maze is to swim in a platform-associated direction guided by exteroceptive and interoceptive cues, a strategy dependent on the ATN. These results also provide further support for the view that the ATN are essential for spatial navigation (Aggleton et al., 1996; Warburton et al., 1997; van Groen et al., 2002; Wilton et al., 2001; Wolff et al., 2008).

The primary behavioral correlate of the firing of ATN neurons is head direction (Taube, 1995), and the ATN play a significant role in the generation of HD cell activity in other limbic cortical 
regions (Taube, 2007). The activity of ATN HD cells have been proposed to be a critical component of the neural network supporting spatial navigation (Taube, 1998), a view supported by the current study. However, reviews of studies in which HD cells were recorded while the rat performed some spatial task indicate little support for this notion (Muir and Taube, 2002; Stackman, 2010). One problem with the recording during spatial behavior approach is that there is often little attention paid to whether the task performed engages the brain structure from which the HD cells are recorded. Therefore, one strategy for establishing the relationship of HD cell activity to spatial navigation would be to first find a task in which the rodent navigates in a particular direction relative to cues, then to test whether such behavioral responding is dependent on the anterior thalamus, and finally to test whether the behavior is dependent on HD cells.

Although the current findings indicate that temporary ATN inactivation disrupts relative responding of mice in the MWM, there are possible alternative interpretations. Lesions of the ATN abolish directional firing of rat postsubiculum neurons (Goodridge and Taube, 1997), suggesting that ATN HD cells are essential for the generation of the directional signal in cortical regions-the postsubiculum and retrosplenial cortex (RSP). However, this interpretation is complicated by evidence that ATN lesions cause "covert pathology" in the RSP (Jenkins et al., 2004), and RSP lesions disrupt the influence of visual landmarks on ATN HD cells (Clark et al., 2010). While, the intrathalamic injection of muscimol diminishes the likelihood of covert RSP pathology, muscimol would have indiscriminately inactivated both directional ( $\sim 60 \%$ of the total number) and nondirectional neurons of the anterior thalamus. Therefore, it is important to consider that the disruption of relative responding after ATN inactivation might be a result of altered activity in the network of brain regions functionally and reciprocally connected with the ATN, such as the RSP and postsubiculum.

To distinguish ATN and CAl contributions to relative responding, a separate cohort of mice received bilateral microinfusion of muscimol into the CA1 region of the dorsal hippocampus. These mice continued to swim first to the relative search zone, albeit with considerably less direct paths. This result suggests dissociation of the influences of the ATN and the hippocampus in guiding spatial search behavior during a probe test in the water maze. This result is interesting since permanent hippocampal lesions impair directional learning of rats in a T-maze task (Stringer et al., 2005) and impair path integration of rats in an open-field homing task (Maaswinkel et al., 1999). The discrepancy in results here may be related to our use of temporary inactivation in mice already trained in the spatial task. It is possible that the intrahippocampal infusion of muscimol did not affect enough of the CA1 region to significantly disrupt relative responding. However, this interpretation is unlikely given that the intrahippocampal muscimol-treated mice failed to swim in direct paths. Collectively, it can be concluded that the ATN guide the notable preference of the mice for relative responding.

Pearce et al. (1998) demonstrated that hippocampal lesions spared spatial learning by rats in a heading vector water maze task. The Pearce et al. (1998) task required the rats to learn to find the submerged platform a fixed direction and distance from a local landmark cue in the pool. Interestingly, lesions of the ATN impaired spatial learning in the Pearce et al. (1998) heading vector task (Wilton et al., 2001). These results indicate a distinction in the degree to which ATN and the hippocampus influence spatial navigation. The hippocampus is critical for the successful encoding of an internal map of the relationships between several distal cues and the goal location (Eichenbaum et al., 1990, 1999; Bird and Burgess, 2008). Compromising hippocampal function disrupts the efficiency with which the rodent can access the internal map or generate an efficient escape trajectory to the goal from a given local view. From the present results, it appears that relative responding is intact after dorsal hippocampal inactivation, a result that is consistent with the results of Pearce et al. (1998). Collectively, the remarkable preference for mice to exhibit relative responding in the MWM despite prior place training is consistent with the view that navigation, and the firing of hippocampal place cells, is influenced by both distal room-based and local or apparatus-based reference frames (Knierim and Hamilton, 2011).

In the present study, when the distal cues were rotated and the pool translated, the relative responding of mice shifted by a corresponding amount. This result provides a clear indication of the dual influence of distal and local cues on platform search behavior in mice. Interestingly, when the ATN were inactivated, the preference for relative responding gave way to absolute responding. Presumably, the ATN inactivation disrupted the contribution of the HD cell network to spatial navigation, thereby disconnecting the medial entorhinal cortex grid cell network from the HD cell system. It is possible that this disconnection is manifested in the tight circling paths and delayed arrival in the absolute zone by the ATN-inactivated mice. In this circumstance, spatial behavior would be guided by altered input to the hippocampus. Support for this view comes from the strategy of the ATN-inactivated mice to shift from relational responding to absolute responding. Thus, manipulations that affect the internal directional sense, such as disorientation or ATN inactivation, impair relative responding and, perhaps, require the rodents to confirm their directional heading and current location by referencing the distal extramaze cues. This view is also supported by evidence that lesions of ATN disrupt the direction-independent firing quality of hippocampal place cells (Calton et al., 2003). Conversely, the results of the CA1 inactivation may reflect aberrant search behavior in mice caused by a failure of hippocampal neurons to accurately represent context-specific spatial relationships that require integration of inputs from medial entorhinal cortex, as proposed previously (Knierim and Hamilton, 2011).

In conclusion, the present study demonstrates a critical role for the ATN in guiding relative responding of $\mathrm{C} 57 \mathrm{BL} / 6 \mathrm{~J}$ mice in the MWM and suggests that such responding is not dependent on the dorsal hippocampus. Considering the theory that the rodent HD cell network represents the directional sense and contributes to a broader network that supports spatial navigation, it will be of interest to determine whether HD cells in the ATN specifically guide relative responding of rodents in spatial tasks. Given evidence that mice may approach spatial problems using strategies that are distinct from rats (Whishaw, 1995; Whishaw and Tomie, 1996), the present results provide important evidence that mice exhibit a rat-like preference for relative responding in the MWM that is dependent on the ATN.

\section{References}

Aggleton JP, Hunt PR, Nagle S, Neave N (1996) The effects of selective lesions within the anterior thalamic nuclei on spatial memory in the rat. Behav Brain Res 81:189-198.

Allen TA, Narayanan NS, Kholodar-Smith DB, Zhao Y, Laubach M, Brown TH (2008) Imaging the spread of reversible brain inactivations using fluorescent muscimol. J Neurosci Methods 171:30-38.

Béracochéa DJ, Jaffard R (1994) Effects of anterior thalamic lesions on spatial memory in mice. Neuroreport 5:917-920. 
Bird CM, Burgess N (2008) The hippocampus and memory: insights from spatial processing. Nat Rev Neurosci 9:182-194.

Blodgett HC, McCutchan K, Mathews R (1949) Spatial learning in the Tmaze: the influence of direction, turn, and food location. J Exp Psychol 39:800-809.

Calton JL, Stackman RW, Goodridge JP, Archey WB, Dudchenko PA, Taube JS (2003) Hippocampal place cell instability after lesions of the head direction cell network. J Neurosci 23:9719-9731.

Clark BJ, Bassett JP, Wang SS, Taube JS (2010) Impaired head direction cell representation in the anterodorsal thalamus after lesions of the retrosplenial cortex. J Neurosci 30:5289-5302.

DeCoteau WE, Hoang L, Huff L, Stone A, Kesner RP (2004) Effects of hippocampus and medial caudate nucleus lesions on memory for direction information in rats. Behav Neurosci 118:540-545.

Eichenbaum H, Stewart C, Morris RG (1990) Hippocampal representation in place learning. J Neurosci 10:3531-3542.

Eichenbaum H, Dudchenko P, Wood E, Shapiro M, Tanila H (1999) The hippocampus, memory, and place cells: is it spatial memory or a memory space? Neuron 23:209-226.

Franklin KBJ, Paxinos G (2008) The mouse brain in stereotaxic coordinates. San Diego: Academic.

Golob EJ, Taube JS (1997) Head direction cells and episodic spatial information in rats without a hippocampus. Proc Natl Acad Sci U S A 94:7645-7650.

Goodridge JP, Taube JS (1997) Interaction between the postsubiculum and anterior thalamus in the generation of head direction cell activity. J Neurosci 17:9315-9330.

Hamilton DA, Akers KG, Weisend MP, Sutherland RJ (2007) How do room and apparatus cues control navigation in the Morris water task? Evidence for distinct contributions to a movement vector. J Exp Psychol Anim Behav Process 33:100-114.

Hamilton DA, Akers KG, Johnson TE, Rice JP, Candelaria FT, Sutherland RJ, Weisend MP, Redhead ES (2008) The relative influence of place and direction in the Morris water task. J Exp Psychol Anim Behav Process 34:31-53.

Hamilton DA, Akers KG, Johnson TE, Rice JP, Candelaria FT, Redhead ES (2009) Evidence for a shift from place navigation to directional responding in one variant of the Morris water task. J Exp Psychol Anim Behav Process 35:271-278.

Jenkins TA, Vann SD, Amin E, Aggleton JP (2004) Anterior thalamic lesions stop immediate early gene activation in selective laminae of the retrosplenial cortex: evidence of covert pathology in rats? Eur J Neurosci 19:3291-3304.

Knierim JJ, Hamilton DA (2011) Framing spatial cognition: neural representations of proximal and distal frames of reference and their roles in navigation. Physiol Rev 91:1245-1279.

Maaswinkel H, Jarrard LE, Whishaw IQ (1999) Hippocampectomized rats are impaired in homing by path integration. Hippocampus 9:553-561.

McNaughton BL, Battaglia FP, Jensen O, Moser EI, Moser MB (2006) Path integration and the neural basis of the "cognitive map." Nat Rev Neurosci 7:663-678.

Mitchell AS, Dalrymple-Alford JC (2006) Lateral and anterior thalamic lesions impair independent memory systems. Learn Mem 13:388-396.

Morris RGM, Garrud P, Rawlins JN, O'Keefe J (1982) Place navigation impaired in rats with hippocampal lesions. Nature 297:681-683.

Moser EI, Kropff E, Moser MB (2008) Place cells, grid cells, and the brain's spatial representation system. Annu Rev Neurosci 31:69-89.

Muir GM, Taube JS (2002) The neural correlates of navigation: do head direction and place cells guide spatial behavior? Behav Cogn Neurosci Rev $1: 297-317$.
O'Keefe J, Nadel L (1978) The hippocampus as a cognitive map. Oxford, UK: Clarendon.

O'Keefe J, Burgess N, Donnett JG, Jeffery KJ, Maguire EA (1998) Place cells, navigational accuracy, and the human hippocampus. Philos Trans R Soc Lond B Biol Sci 353:1333-1340.

Pearce JM, Roberts AD, Good M (1998) Hippocampal lesions disrupt navigation based on cognitive maps but not heading vectors. Nature 396:75-77.

Potegal M, Day MJ, Abraham L (1977) Maze orientation, visual and vestibular cues in two-maze spontaneous alternation of rats. Physiol Psychol $5: 414-420$.

Semenov LV, Bures J (1989) Vestibular stimulation disrupts acquisition of place navigation in the Morris water tank task. Behav Neural Biol 51:346-363.

Skinner DM, Etchegary CM, Ekert-Maret EC, Baker CJ, Harley CW, Evans JH, Martin GM (2003) An analysis of response, direction, and place learning in an open field and T maze. J Exp Psychol Anim Behav Process 29:3-13.

Stackman RW (2010) Behavioral correlates of neuronal activity recorded as single-units. Promises and pitfalls as illustrated by the rodent head direction cell signal. In: Electrophysiological recording methods (Vertes RP, Stackman RW Jr, eds). New York: Springer.

Stackman RW, Herbert AM (2002) Rats with lesions of the vestibular system require a visual landmark for spatial navigation. Behav Brain Res 128:27-40.

Stringer KG, Martin GM, Skinner DM (2005) The effects of hippocampal lesions on response, direction, and place learning in rats. Behav Neurosci 119:946-952.

Taube JS (1995) Head direction cells recorded in the anterior thalamic nuclei of freely moving rats. J Neurosci 15:70-86.

Taube JS (1998) Head direction cells and the neurophysiological basis for a sense of direction. Prog Neurobiol 55:225-256.

Taube JS (2007) The head direction signal: origins and sensory-motor integration. Annu Rev Neurosci 30:181-207.

Tsanov M, Chah E, Vann SD, Reilly RB, Erichsen JT, Aggleton JP, O’Mara SM (2011) Theta-modulated head direction cells in the rat anterior thalamus. J Neurosci 31:9489-9502.

van Groen T, Kadish I, Michael Wyss J (2002) Role of the anterodorsal and anteroventral nuclei of the thalamus in spatial memory in the rat. Behav Brain Res 132:19-28.

Wallace DG, Hines DJ, Pellis SM, Whishaw IQ (2002) Vestibular information is required for dead reckoning in the rat. J Neurosci 22:10009-10017.

Warburton EC, Baird AL, Aggleton JP (1997) Assessing the magnitude of the allocentric spatial deficit associated with complete loss of the anterior thalamic nuclei in rats. Behav Brain Res 87:223-232.

Whishaw IQ (1995) A comparison of rats and mice in a swimming pool place task and matching to place task: some surprising differences. Physiol Behav 58:687-693.

Whishaw IQ, Tomie J (1996) Of mice and mazes: similarities between mice and rats on dry land but not water mazes. Physiol Behav 60:1191-1197.

Wilton LA, Baird AL, Muir JL, Honey RC, Aggleton JP (2001) Loss of the thalamic nuclei for "head direction" impairs performance on spatial memory tasks in rats. Behav Neurosci 115:861-869.

Wolff M, Gibb SJ, Cassel JC, Dalrymple-Alford JC (2008) Anterior but not intralaminar thalamic nuclei support allocentric spatial memory. Neurobiol Learn Mem 90:71-80.

Yoganarasimha D, Yu X, Knierim JJ (2006) Head direction cell representations maintain internal coherence during conflicting proximal and distal cue rotations: comparison with hippocampal place cells. J Neurosci 26: $622-631$ 